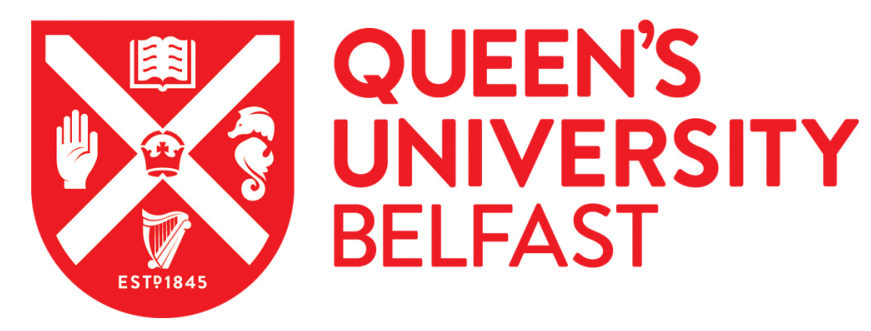

\title{
MCC950 directly targets the NLRP3 ATP-hydrolysis motif for inflammasome inhibition
}

\author{
Coll, R. C., Hill, J. R., Day, C. J., Zamoshnikova, A., Boucher, D., Massey, N. L., Chitty, J. L., Fraser, J. A., \\ Jennings, M. P., Robertson, A. A. B., \& Schroder, K. (2019). MCC950 directly targets the NLRP3 ATP-hydrolysis \\ motif for inflammasome inhibition. Nature Chemical Biology, 15, 556-559. https://doi.org/10.1038/s41589-019- \\ 0277-7
}

Published in:

Nature Chemical Biology

Document Version:

Peer reviewed version

Queen's University Belfast - Research Portal:

Link to publication record in Queen's University Belfast Research Portal

Publisher rights

(c) The Author(s), under exclusive licence to Springer Nature America, Inc. 2019.

This work is made available online in accordance with the publisher's policies. Please refer to any applicable terms of use of the publisher.

\section{General rights}

Copyright for the publications made accessible via the Queen's University Belfast Research Portal is retained by the author(s) and / or other copyright owners and it is a condition of accessing these publications that users recognise and abide by the legal requirements associated with these rights.

Take down policy

The Research Portal is Queen's institutional repository that provides access to Queen's research output. Every effort has been made to ensure that content in the Research Portal does not infringe any person's rights, or applicable UK laws. If you discover content in the Research Portal that you believe breaches copyright or violates any law, please contact openaccess@qub.ac.uk. 


\title{
Nature Chemical Biology: Brief Communication
}

Title: MCC950 directly targets the NLRP3 ATP hydrolysis motif for inflammasome inhibition

Authors: Rebecca C. Coll ${ }^{1}$, James R. Hill ${ }^{1}$, Christopher J. Day ${ }^{2}$, Alina Zamoshnikova $^{1,3}$, Dave Boucher ${ }^{1,4}$, Nicholas L. Massey ${ }^{1}$, Jessica L. Chitty, ${ }^{5,6,7}$, James A. Fraser ${ }^{5}$, Michael P. Jennings ${ }^{2}$, Avril A. B. Robertson ${ }^{1,8 *}$, Kate Schroder ${ }^{1,5 *}$

\begin{abstract}
Affiliations:
${ }^{1}$ Institute for Molecular Bioscience and IMB Centre for Inflammation and Disease Research, The University of Queensland, St Lucia, Queensland, Australia.

2Institute for Glycomics, Griffith University, Gold Coast, Queensland, Australia.

${ }^{3}$ Current address: The University of Queensland Diamantina Institute, Translational

Research Institute, Brisbane, Queensland, Australia.

${ }^{4}$ Current address: Department of Biochemistry, University of Lausanne, Epalinges

$\mathrm{CH} 1066$, Switzerland.

${ }^{5}$ Australian Infectious Diseases Research Centre, School of Chemistry and

Molecular Biosciences, The University of Queensland, St Lucia, Queensland,

Australia.

${ }^{6}$ Current address: The Garvan Institute of Medical Research \& the Kinghorn Cancer Centre, Cancer Division, Sydney, New South Wales, Australia.

${ }^{7}$ Current address: St Vincent's Clinical School, Faculty of Medicine, UNSW Sydney, New South Wales, Australia

${ }^{8}$ School of Chemistry and Molecular Biosciences, The University of Queensland, St Lucia, Queensland, Australia.
\end{abstract}

*Joint senior authors

Correspondence should be addressed to K.S. (k.schroder@imb.uq.edu.au), R.C.C. (r.coll@imb.uq.edu.au) or A.A.B.R (a.robertson3@uq.edu.au)

\begin{abstract}
:
Inhibition of the NLRP3 inflammasome is a promising strategy for the development of new treatments for inflammatory diseases. MCC950 is a potent and specific small-molecule inhibitor of the NLRP3 pathway, but its molecular target is not defined. Here we show that MCC950 directly interacts with the Walker B motif within the NLRP3 NACHT domain, thereby blocking ATP hydrolysis and inhibiting NLRP3 activation and inflammasome formation.
\end{abstract}

\section{Main Text:}

The NOD-like receptor (NLR) family, pyrin domain-containing protein 3 (NLRP3) is a cytosolic sensor of diverse pathogen- and host-derived molecules. Upon activation, NLRP3 oligomerises and recruits apoptosis-associated speck-like protein containing a CARD (ASC), forming a platform for the binding, dimerisation and activation of the caspase-1 protease ${ }^{1}$. Caspase- 1 then cleaves the pro-inflammatory cytokines prointerleukin-1 $\beta$ (IL-1 $\beta$ ) and pro-IL-18, mediating the secretion of their active cytokines. Caspase-1 also cleaves Gasdermin-D, triggering pyroptosis ${ }^{2,3}$. NLRP3-driven inflammation is pathological in the development of many diseases including cryopyrin-associated periodic syndromes, Alzheimer's Disease, Parkinson's 
Disease, gout, atherosclerosis, non-alcoholic fatty liver disease, asthma, and silicosis $^{4-6}$. Inhibitors of NLRP3 are thus potential treatments for these conditions with unmet clinical needs.

We previously described MCC950 (CP-456,773, CRID3), a potent and specific small molecule inhibitor of the NLRP3 inflammasome ${ }^{7}$. MCC950 has since been validated in vivo in numerous species and disease models ${ }^{8-10}$, and is a useful tool molecule for the field ${ }^{11,12}$. It is important to identify the mechanism of action (MoA) and molecular target of a small molecule to inform and de-risk drug development ${ }^{13}$. We reported that MCC950 did not suppress NLRP3 activity via effects on inflammasome priming, calcium signalling, potassium efflux, or NLRP3-ASC interaction ${ }^{7}$. Other studies demonstrated that MCC950 inhibits potassium efflux-independent NLRP3 activation ${ }^{14,15}$ but does not block mitochondrial respiration or reactive oxygen species production ${ }^{14}$. NLRP3 activation requires interaction with NEK $7^{16}$, and MCC950 is postulated to target NEK7 to block NLRP3 activation ${ }^{3,17}$. Though the capacity of MCC950 to block NLRP3 signalling is well established, we sought to identify the molecular target of MCC950 and more precisely delineate its MoA.

MCC950 specifically inhibits NLRP3 amongst inflammasomes ${ }^{7,18}$, and blocks canonical, non-canonical, and alternative NLRP3 activation ${ }^{7,14,15}$. We thus hypothesised that MCC950 directly inhibits NLRP3. We initially tested this using a drug affinity responsive target stability (DARTS) approach ${ }^{19}$, using mouse bone marrow-derived macrophages (BMM), and the broad specificity protease mix, pronase. The assay was optimised (Supplementary Fig. 1) to induce degradation of NLRP3, as indicated by immunoblot detection of numerous pronase-induced NLRP3 fragments. Increasing doses of MCC950 (0.1-10 $\mu \mathrm{M})$ protected NLRP3 from pronase-mediated degradation, as evident from the changes in digestion pattern detected using antibodies against the NACHT or PYD domains (Fig. 1a). The effect of MCC950 was specific to NLRP3; MCC950 did not block the degradation of NEK7 or GAPDH at the optimised pronase concentration (Fig. 1a) or a lower pronase concentration (Supplementary Figs. 1, 2a). MCC950 similarly limited NLRP3 protein degradation in primary human monocyte-derived macrophages (Supplementary Fig. 2b), and by an alternative protease, thermolysin (Supplementary Fig. 2c). We next examined whether NLRP3 activation status affected MCC950-dependent protease protection. We performed DARTS assays using LPS-primed BMM stimulated with the NLRP3 activator, nigericin, and then treated with MCC950, and compared this to MCC950 exposure to unstimulated or single-stimulated controls in which NLRP3 is not activated. Pycard ${ }^{-/-}\left(\right.$Asc $\left.^{-/-}\right)$BMM were used to prevent inflammasome-mediated cell death. MCC950 protected NLRP3 from degradation in all conditions (Supplementary Fig. 2d). This suggests that MCC950 directly interacts with NLRP3 in both its inactive and active conformations.

To verify that MCC950 interacts with NLRP3, we used a photo-affinity labelling strategy. Based on the structure of MCC950, we synthesised a benzophenone and alkyne-containing photoaffinity probe (PAP, 1) that inhibits NLRP3 activation (Supplementary Fig. 3), and covalently cross-links to its molecular target when activated by ultraviolet (UV) light. Recombinant human NLRP3 without the leucine rich repeat (LRR) domain (NLRP3 $\triangle L R R$, Fig. 1b, Supplementary Fig. 4) and BMM lysates (Fig. 1C) were treated with the PAP, in the presence or absence of MCC950 to compete for binding. Following UV exposure, the PAP was linked to biotin-azide 
via $\mathrm{Cu}(\mathrm{I})$-catalysed click chemistry, and purified using streptavidin magnetic particles. The biotinylated PAP indeed pulled down recombinant NLRP3 $\triangle L R R$ (Fig. 1 b) and NLRP3 from BMM lysates (Fig. 1c), and these interactions were suppressed by MCC950 competition (Fig. 1b-c), confirming that MCC950 directly interacts with NLRP3.

To identify the NLRP3 domain that binds to MCC950, we expressed full-length NLRP3 versus truncation mutants (Fig. 2a) in HEK293T cells, and performed DARTS assays (Fig. 2b-d). MCC950 protected full-length and $\triangle$ LRR NLRP3 (Fig. 2b-c; see arrows), but not the NLRP3 PYD domain (Fig. 2d) from degradation, suggesting that MCC950 binds to the central NACHT domain. The NLRP12 NACHT is the most closely related to the NLRP3 NACHT within the NLR family ${ }^{20}$. A chimeric form of NLRP3 ('NACHT12'), in which the NACHT is swapped for that of NLRP12, was not protected by MCC950 in a DARTS assay (Supplementary Fig. 5). This indicates that MCC950 specifically interacts with the NLRP3 NACHT domain.

We next sought to identify the MCC950-interaction site within the NACHT domain. The D4D8T antibody clone was raised against residues around Alanine 306 within the NLRP3 NACHT domain (Supplementary Fig. 6a). We used this antibody to immunoprecipitate NLRP3 in LPS-primed BMM (Fig. 3a). MCC950 dosedependently decreased the efficiency of NLRP3 immunoprecipitation, suggesting that MCC950 interacts with NLRP3 at a site close to the epitope of this antibody and thereby blocks antibody:NLRP3 interactions. MCC950 also suppressed D4D8Tmediated, but not anti-mCherry antibody-mediated, immunoprecipitation of mCherrytagged NLRP3 (Supplementary Fig. 6b), indicating that the effect of MCC950 is specific for the D4D8T antibody. Immunoprecipitation assays were also performed with lysates from Asc $^{-/}$BMM under conditions where NLRP3 is active (LPS plus nigericin) or inactive (single stimulated or untreated controls). MCC950 blocked D4D8T-mediated NLRP3 immunoprecipitation in all conditions (Fig. 3b), validating previous observations from DARTS assays (Supplementary Fig. 2d) that MCC950 interacts with both active and inactive NLRP3.

Alanine 306 of mouse NLRP3 is located near the ATP binding (Walker A) and hydrolysis (Walker B) motifs (Supplementary Fig. 6a). To define the D4D8T antibody epitope, we tested its ability to detect NLRP3 Walker A and Walker B motif mutants, or the NACHT12 chimera (Supplementary Fig. 6c). The D4D8T antibody failed to recognise the NLRP12 NACHT, and poorly detected NLRP3 mutants in which the key Walker B motifs are substituted (Walker B single or A+B double mutants) while the NLRP3 Walker A single mutant was readily detected. As this suggested that the D4D8T antibody binds to NLRP3 at the Walker B motif, we next used DARTS assays to determine whether Walker B mutation disrupts MCC950 binding. Immunoblotting revealed that while MCC950 prevented the degradation of wild-type NLRP3 and the inactive Walker A mutant (Fig. 3c, Supplementary Fig. 6d; see arrows), MCC950 did not protect the Walker B or Walker A+B mutants from protease degradation (Fig. 3d, Supplementary Fig. 6e). These data indicate that MCC950 interacts with NLRP3 proximal to the Walker B motif.

The kinetics of MCC950 activity, and whether it is a covalent NLRP3 inhibitor, is unknown. In LPS-primed BMM, MCC950 potently inhibits nigericin-induced IL-1 $\beta$ release and cell death (Fig. 3e, Supplementary Fig. 7). When MCC950 was 
washed out prior to nigericin treatment, however, its potency markedly decreased (Fig. 3e, Supplementary Fig. 7), with a shift in $\mathrm{IC}_{50}$ from approximately $24 \mathrm{nM}$ to 1.7 $\mu \mathrm{M}$. To further establish MCC950:NLRP3 interaction kinetics, we performed surface plasmon resonance with immobilised recombinant NLRP3 2 LRR. MCC950 binds to NLRP3 $\triangle L R R$ with high affinity $\left(K_{D}=224 \mathrm{nM}\right)$, and a rapid off-rate $\left(k_{d}=0.247 \mathrm{~s}^{-1}\right)$ (Fig. 3f, Supplementary Table 1), supporting our observation that MCC950 can be readily washed out in cell-based assays (Fig. 3e). Thus, MCC950 is a reversible NLRP3 inhibitor.

We next sought to determine the impact of MCC950 interaction on NLRP3 molecular function. Given that MCC950 binds proximal to the Walker B motif that mediates ATP hydrolysis (Fig. 3a-d), and ATP hydrolysis is required for NLRP3 inflammasome assembly and function ${ }^{21}$, we hypothesised that MCC950 may inhibit NLRP3 by suppressing its ATPase activity. ATP indeed binds to NLRP3 $L$ LRR $\left(K_{D}=2.05 \mu \mathrm{M}\right)$, with an off-rate $\left(\mathrm{k}_{\mathrm{d}}=24.15 \mathrm{~s}^{-1}\right)$ consistent with ATP hydrolysis by NLRP3 (Fig. 3f, Supplementary Table 1). When NLRP3 $\triangle$ LRR was exposed to both MCC950 and ATP, these two molecules did not compete for NLRP3 $\triangle$ LRR binding (Fig. 3f), but remarkably, led to high affinity, stable interaction $\left(K_{D}=10.6 \mathrm{nM}, \mathrm{K}_{\mathrm{d}}=1.47 \times 10^{-3} \mathrm{~s}^{-1}\right.$; Fig. 3f, Supplementary Table 1). In contrast, ADP binds poorly to NLRP3 $\Delta L R R$, and its combination with MCC950 does not induce a high affinity, stable interaction (Fig. 3g, Supplementary Table 1). The NLRP3 $\triangle$ LRR Walker B mutant did not interact with MCC950 (Fig. 3h, Supplementary Fig. 8a, Supplementary Table 1), but did bind ATP, as is anticipated from the intact NLRP3 Walker A site. Confirming earlier observations, recombinant NEK7 did not bind MCC950 (Supplementary Fig. 8b-c, Supplementary Table 1), but did interact with ATP, as expected from NEK7 kinase function. Altogether, these data indicate that MCC950 binds proximal to the NLRP3 Walker B motif and prevents NLRP3 from hydrolysing ATP to ADP.

In summary, we reveal the molecular mechanism of action of MCC950. MCC950 specifically binds to both active and inactive NLRP3, in a high-affinity non-covalent interaction at or adjacent to the Walker B motif, thereby blocking the ability of NLRP3 to hydrolyse ATP for NLRP3 inflammasome function. Our data confirm the critical role of ATP binding and hydrolysis to NLRP3 function ${ }^{21}$, consistent with the known ATP-dependent oligomerisation mechanisms of NLRC4 ${ }^{22,23}$ and other $\mathrm{AAA}^{+}$ ATPases $^{24}$. The accompanying study by Tapia-Abellán et al. ${ }^{25}$ reports that NLRP3 undergoes structural rearrangements to open out during activation, and that MCC950 counters this via interaction with the NLRP3 Walker B site, driving NLRP3 towards a closed and inactive conformation. These complementary studies together show that MCC950 binds to NLRP3 and prevents it from assuming or retaining its active open conformation, and thereby blocks NLRP3 oligomerisation and inflammasome function. They further suggest that ATP hydrolysis is required not only for NLRP3 to assume its open conformation during activation, but also for maintaining this active conformer. Our elucidation of MCC950 mechanism of action paves the way for further rational development of small-molecule inhibitors to treat NLRP3-driven inflammatory diseases.

\section{Acknowledgements:}

This work was supported by the National Health and Medical Research Council of Australia (Fellowship 1138466 and Program Grant 1071659 to MPJ, 
Fellowship 1141131 to KS, Project Grant 1086786 to AABR and KS), the Australian Research Council (Fellowship FT130100361 to KS), the Institute for Molecular Bioscience (Research Advancement Award to $\mathrm{JH}$ ) and The University of Queensland (Postdoctoral Fellowships to RCC and DB, Research Scholarship to JH). We thank David Edwards for chemical purification and analytical support, Professor Matthew Cooper (University of Queensland) for providing MCC950, and Associate Professor Katryn Stacey (University of Queensland) for providing ASC-deficient mice.

\section{Author Contributions:}

R.C.C. designed and performed most experiments. J.R.H. conceived and synthesised the photoaffinity probe and performed labelling experiments. C.J.D. and M.P.J. designed and performed surface plasmon resonance analysis. A.Z. designed and cloned the NLRP3 expression plasmids and mutants. D.B. expressed and purified recombinant NLRP3 and assisted with experimental design. N.L.M synthesised the photoaffinity probe. J.L.C. and J.A.F. assisted with the expression and purification of recombinant NEK7, A.A.B.R. formulated MCC950, conceived the photoaffinity probe, and expressed and purified recombinant NEK7. A.A.B.R and K.S. designed experiments and supervised the study. R.C.C and K.S wrote the manuscript, with assistance from J.R.H. and A.A.B.R., and input from all authors.

\section{Competing Financial Interests:}

RC, AABR, and KS are co-inventors on patent applications for NLRP3 inhibitors (WO2018215818, WO2017140778 and WO2016131098) which are licensed to Inflazome Ltd, a company headquartered in Dublin, Ireland. Inflazome is developing drugs that target the NLRP3 inflammasome to address unmet clinical needs in inflammatory disease.

\section{Data Availability:}

The data that support the findings of this study are available from the corresponding author upon reasonable request. 


\section{References}

1. $\quad$ Boucher, D., et al. J Exp Med 215, 827-840 (2018).

2. $\quad$ Broz, P. \& Dixit, V.M. Nat Rev Immunol 16, 407-420 (2016).

3. Prochnicki, T., Mangan, M.S. \& Latz, E. F1000Res 5(2016).

4. Guo, H., Callaway, J.B. \& Ting, J.P. Nat Med 21, 677-687 (2015).

5. $\quad$ Broderick, L., et al. Annu Rev Pathol 10, 395-424 (2015).

6. De Nardo, D., De Nardo, C.M. \& Latz, E. Am J Pathol 184, 42-54 (2014).

7. Coll, R.C., et al. Nat Med 21, 248-255 (2015).

8. van Hout, G.P., et al. Eur Heart J 38, 828-836 (2017).

9. $\quad$ Primiano, M.J., et al. J Immunol 197, 2421-2433 (2016).

10. Kim, R.Y., et al. Am J Respir Crit Care Med 196, 283-297 (2017).

11. Tate, M.D., et al. Sci Rep 6, 27912 (2016).

12. Kammoun, H.L., et al. Mol Metab (2018).

13. Moffat, J.G., et al. Nat Rev Drug Discov 16, 531-543 (2017).

14. Gross, C.J., et al. Immunity 45, 761-773 (2016).

15. Gaidt, M.M., et al. Immunity 44, 833-846 (2016).

16. Shi, H., et al. Nat Immunol 17, 250-258 (2016).

17. White, C.S., Lawrence, C.B., Brough, D. \& Rivers-Auty, J. Brain Pathol 27, 223-234 (2017).

18. Van Gorp, H., et al. P Natl Acad Sci USA 113, 14384-14389 (2016).

19. Lomenick, B., et al. P Natl Acad Sci USA 106, 21984-21989 (2009).

20. Schroder, K. \& Tschopp, J. Cell 140, 821-832 (2010).

21. Duncan, J.A., et al. P Natl Acad Sci USA 104, 8041-8046 (2007).

22. Hu, Z., et al. Science 341, 172-175 (2013).

23. Tenthorey, J.L., et al. Science 358, 888-893 (2017).

24. Wendler, P., Ciniawsky, S., Kock, M. \& Kube, S. Biochim Biophys Acta 1823, 2-14 (2012).

25. Tapia-Abellán, A., et al. Nat Chem Biol, In press (\#NCHEMB-BC180708014). 


\section{Figure Legends}

Figure 1. MCC950 directly interacts with NLRP3. (a) LPS-primed BMM were treated with MCC950 (0.1-10 $\mu \mathrm{M})$ or vehicle for $1 \mathrm{~h}$, cells were lysed in buffer +/MCC950 (0.1-10 $\mu \mathrm{M})$. DARTS assay was performed with pronase $(500 \mathrm{ng} / \mathrm{\mu g}$ of protein) and lysates analysed by immunoblot using antibodies for NLRP3 PYD (cryo2), NLRP3 NACHT (D4D8T), NEK7 and GAPDH. (b) Recombinant human NLRP3 $\triangle L R R$ or (c) LPS-primed BMM lysates were treated with $5 \mu \mathrm{M}$ MCC950 or vehicle control. Samples were then treated with $5 \mu \mathrm{M}$ PAP and exposed to $20 \mathrm{~W} 365$ $\mathrm{nm}$ UV light for 90 min. Biotinylation reagents were added and incubated at RT for $60 \mathrm{~min}$, or biotin was omitted as a control. Total protein was precipitated and samples were resuspended and incubated with streptavidin paramagnetic particles. Interacting proteins were analysed by immunoblot. Data are representative of (a) $\mathrm{N}=5$ or $(\mathbf{b}, \mathbf{c}) \mathrm{N}=2$ independent experiments. Uncropped Western blots are presented in Supplementary Figure 9.

Figure 2. MCC950 interacts with the NACHT domain of NLRP3. (a) Schematic diagram of the NLRP3 domain truncations used in b-d. (b-d) HEK293T cells transfected with mCherry-tagged full-length (FL) NLRP3 (b), NLRP3 $L$ LRR (c), or NLRP3-PYD (d), were lysed in buffer +/- MCC950 $(10 \mu \mathrm{M})$. DARTS assays were performed with pronase $(200 \mathrm{ng} / \mu \mathrm{g}$ of protein) and lysates were analysed by immunoblot using antibodies for NLRP3 PYD, NLRP3 NACHT and GAPDH. (b-c) Arrows indicate changes in degradation observed with MCC950 treatment. Data are representative of $\mathrm{N}=4$ independent experiments. Uncropped Western blots are presented in Supplementary Figure 10.

Figure 3. MCC950 binds non-covalently to NLRP3, proximal to the Walker $B$ motif, and blocks NLRP3 ATPase activity (a) LPS-primed C57BL/6 BMM and (b) Asc ${ }^{-1-}$ BMM unstimulated or treated with LPS (4 h) and/or nigericin (30 min), were lysed in buffer +/- MCC950 $(10 \mu \mathrm{M})$ and immunoprecipitation (IP) of NLRP3 was performed with the NLRP3 NACHT D4D8T antibody. NLRP3 levels in the input and IP samples were analysed by immunoblot using antibodies for NLRP3 PYD (cryo-2) and GAPDH. (c-d) HEK293T cells transfected with mCherry-tagged WT NLRP3 (c), or Walker B mutant (d) for $24 \mathrm{~h}$. Cells were lysed in buffer +/- MCC950 $(10 \mu \mathrm{M})$. DARTS assays were performed with pronase $(200 \mathrm{ng} / \mu \mathrm{g}$ of protein) and lysates analysed by immunoblot using antibodies for NLRP3 PYD (cryo-2), NLRP3 NACHT (D4D8T), and GAPDH. (c) Arrows indicate changes in degradation observed with MCC950 treatment. Data are representative of $(\mathbf{a}, \mathbf{b}) \mathrm{N}=3$, (c) $\mathrm{N}=5$, or (d) $\mathrm{N}=4$ independent experiments. (e) IL-1 $\beta$ secretion from LPS-primed BMM treated with MCC950 $(0.01-10 \mu \mathrm{M})$ in the final 30 min of priming and then stimulated with nigericin, as measured by ELISA. MCC950 was left on cells or washed out for $1 \mathrm{~min}$ prior to nigericin treatment. Cytokine level is normalized to that of vehicle-treated control cells. Data are the mean of $\mathrm{N}=4$ independent experiments, each experiment was performed in technical triplicate, error bars are \pm S.E.M. Nonlinear regression analysis was performed, and the curve of Log [M] MCC950 versus the normalized response (variable slope) is presented. (f-h) Surface plasmon resonance analysis of MCC950, ATP, and ADP interaction with wild-type $(\mathbf{f}, \mathbf{g})$ or Walker B mutant $(\mathbf{h})$ recombinant NLRP3 $\triangle$ LRR. (f-h) Representative sensorgrams are shown from $\mathrm{N}=3$ independent experiments, where protein was loaded twice and three technical 
replicates per run were performed. Uncropped Western blots are presented in Supplementary Figure 11.

\section{Online Methods}

MCC950 synthesis and formulation. MCC950 sodium salt was synthesised as previously described ${ }^{7}$. MCC950 sodium salt stock solutions were prepared in $\mathrm{H}_{2} \mathrm{O}$.

Mouse and human primary macrophage cell culture. All experimental protocols involving mice were approved by the UQ Animal Ethics Committee. Studies using primary human cells were approved by the UQ Human Medical Research Ethics Committee. We have complied with all relevant ethical regulations. C57BL/6 and $A s C^{-1-26}$ mice were housed in specific pathogen-free facilities at the University of Queensland (UQ). The Australian Red Cross Blood Service receives blood donations from informed and consenting healthy adult donors, and provided buffy coats from anonymous donors for this research study. Peripheral blood mononuclear cells were isolated from buffy coats by density centrifugation using Ficoll-Paque Plus (GE Healthcare). CD14 ${ }^{+}$monocytes were then isolated using magnetic-activated cell sorting (Miltenyi Biotech), according to the manufacturer's instructions. Human and murine macrophages were differentiated from human $\mathrm{CD} 14^{+}$monocytes and murine bone marrow as previously described, and were used for experiments on day 7 of differentation ${ }^{27}$. Mouse bone marrow-derived macrophages (BMMs) and human monocyte-derived macrophages (HMDMs) were cultured in media consisting of RPMI 1640 medium (Life Technologies) supplemented with 10\% heat-inactivated fetal calf serum (FCS), 2 mM GlutaMAX (Life Technologies), $50 \mathrm{U} / \mathrm{ml}$ penicillinstreptomycin (Life Technologies) and $150 \mathrm{ng} / \mathrm{ml}$ recombinant human macrophage colony-stimulating factor (CSF-1 [endotoxin free, expressed and purified by the UQ Protein Expression Facility]).

HEK293T cell culture and transfection. HEK293T cells were cultured in DMEM containing high glucose, pyruvate (Life Technologies), and supplemented with 10\% FCS and $50 \mathrm{U} / \mathrm{ml}$ penicillin-streptomycin. HEK293T cells $\left(3 \times 10^{5} / \mathrm{ml}\right)$ were transfected in $10 \mathrm{~cm}$ dishes via the calcium phosphate method with $5 \mu \mathrm{g}$ of plasmid DNA. The mammalian expression vector, pEF6, was used for all plasmids, and contained mouse NLRP3 with a C-terminal mCherry tag: full length wild type NLRP3, NLRP3ALRR, NLRP3 PYD, NLRP3 Walker A mutant (G227A, K228A, T229A), NLRP3 Walker B mutant (D298A, D301A, E302A), NLRP3 Walker A and B double mutant (G227A, K228A, T229A, D298A, D301A, E302A), and NLRP3-NLRP12 $\mathrm{NACHT}$ domain chimera.

Drug Affinity Responsive Target Stability assays. Assays were adapted from published protocols ${ }^{19,28,29}$. Differentiated BMM and HMDM were plated at a density of $5 \times 10^{5} \mathrm{cells} / \mathrm{ml}$ in $10 \mathrm{~cm}$ dishes in full media. The next day media were removed and replaced with Opti-MEM supplemented with CSF-1. Cells were primed with ultrapure Escherichia coli K12 LPS (100 ng/ml) for 4-6 h, and MCC950 (0.1-10 $\mu \mathrm{M})$ was added for the final hour. In some experiments cells were primed with ultrapure E. coli K12 LPS (100 ng/ml) for $4 \mathrm{~h}$ and stimulated with $5 \mu \mathrm{M}$ nigericin (SigmaAldrich) for $30 \mathrm{~min}$ as indicated with no addition of MCC950 to the cells in culture. HEK293T cells were harvested $24 \mathrm{~h}$ after transfection. Media were removed and cells were washed in PBS (+/- MCC950), cells were lysed in lysis buffer (50 mM Tris- 
$\mathrm{HCl} \mathrm{pH}$ 7.4, $150 \mathrm{mM} \mathrm{NaCl}, 1 \mathrm{mM}$ ATP, 2 mM EDTA, 0.5\% Igepal CA-630) containing protease inhibitors (cOmplete mini protease inhibitor cocktail; Roche), benzonase, and MCC950 as indicated. Lysates were disrupted by passage through a 27-gauge needle and cleared by centrifugation at $14,000 \mathrm{G}$ for $10 \mathrm{~min}$ at $4{ }^{\circ} \mathrm{C}$. Protein concentration was determined using a Pierce ${ }^{\mathrm{TM}}$ BCA Protein Assay Kit (Life Technologies), and a minimum of $20 \mu \mathrm{g}$ of protein lysate was used per reaction. Pronase and thermolysin (10 $\mathrm{mg} / \mathrm{ml}$ [Sigma-Aldrich]) were added at the indicated protease to protein ratio, between $50-500 \mathrm{ng}$ Pronase or $0.05-5 \mu \mathrm{g}$ Thermolysin per $\mu \mathrm{g}$ of protein in the sample, for the indicated time $(7.5-30 \mathrm{~min})$ at room temperature (RT). The reaction was stopped by addition of $20 \mathrm{X}$ protease inhibitor cocktail and incubated on ice for $10 \mathrm{~min}$. Protein samples were analysed by immunoblotting.

Photoaffinity labelling and affinity purification of probe-modified proteins. The photoaffinity labelling and purification methodology was adapted from Xu et al. ${ }^{30}$ and MacKinnon and Taunton ${ }^{31}$. BMM were plated at a density of $5 \times 10^{5}$ cells $/ \mathrm{ml}$ in $10 \mathrm{~cm}$ dishes in full media. The next day cells were primed with ultrapure E. coli K12 LPS $(50 \mathrm{ng} / \mathrm{ml})$ for 4-6 h. Media were removed and cells were washed in PBS and then lysed in lysis buffer (50 mM Tris- $\mathrm{HCl} \mathrm{pH}$ 7.4, $150 \mathrm{mM} \mathrm{NaCl}, 1 \mathrm{mM}$ ATP, 0.5\% lgepal CA-630) containing protease inhibitors and benzonase. Lysates were disrupted by passage through a 27-gauge needle and cleared by centrifugation at $14,000 \mathrm{G}$ for 10 min at $4{ }^{\circ} \mathrm{C}$. Clarified lysates $(1 \mathrm{ml})$ were added to a 24 -well tissue culture plate.

Recombinant NLRP3 $\Delta$ LRR was diluted in lysis buffer and $150 \mu \mathrm{l}$ was added to a 48well tissue culture plate. Samples were treated with $5 \mu \mathrm{M}$ PAP plus $5 \mu \mathrm{M}$ MCC950 or $5 \mu \mathrm{M}$ PAP plus vehicle control. Photo-cross-linking was performed for $90 \mathrm{~min}$, mixing after 45 min, using a custom made light box equipped with a UVA fluorescent tube (Sylvania, $20 \mathrm{~W}, 365 \mathrm{~nm}$ ). Sample plates were placed on ice and within $2 \mathrm{~cm}$ of the fluorescent tube. Biotinylation was performed by adding the following reagents, with final concentrations indicated: biotin-PEG3-azide (biotin- $\mathrm{N}_{3} ; 100 \mu \mathrm{M}$ ), tris[(1-benzyl$1 H$-1,2,3-triazol-4-yl)methyl]amine (TBTA; $100 \mu \mathrm{M}$ ), tris(2-carboxyethyl)phosphine hydrochloride (TCEP; $1 \mathrm{mM}$ ) and $\mathrm{CuSO}_{4} .5 \mathrm{H}_{2} \mathrm{O}(1 \mathrm{mM})$, except in the case of the control where biotin- $\mathrm{N}_{3}$ was omitted. Following reagent addition, plates were mixed at $\mathrm{RT}$ for $1 \mathrm{~h}$. Protein samples were then precipitated with acetone and frozen at $-20{ }^{\circ} \mathrm{C}$ overnight. Precipitated protein was pelleted by centrifugation at $17,000 \mathrm{G}$ for $15 \mathrm{~min}$ at $4{ }^{\circ} \mathrm{C}$. Cold acetone precipitation was repeated twice and protein pellets were then solubilised in $1 \%$ SDS in PBS and diluted in affinity purification buffer $(50 \mathrm{mM}$ HEPES pH 7.4,100 mM NaCl, 1\% Triton X-100). Streptavidin MagneSphere paramagnetic particles (Promega) were blocked with 1\% BSA PBS and washed with affinity purification buffer. The protein samples were incubated with streptavidin paramagnetic particles with gentle rotation for $3 \mathrm{~h}$ at $4{ }^{\circ} \mathrm{C}$. Particles were washed twice in affinity purification buffer and four times in wash buffer $(50 \mathrm{mM} \mathrm{HEPES} \mathrm{pH}$ $7.4,500 \mathrm{mM} \mathrm{NaCl}, 1 \%$ Triton X-100). Bound proteins from BMM lysates were eluted by the addition of elution buffer ( $2 \mathrm{mM}$ D-biotin, $1 \% \mathrm{v} / \mathrm{v}$ Triton X-100, PBS) and incubation at $95^{\circ} \mathrm{C}$ for $5 \mathrm{~min}$. Bound protein from the recombinant NLRP $3 \Delta L R R$ experiments were eluted by boiling particles for $5 \mathrm{~min}$ in affinity purification buffer containing NuPAGE LDS sample buffer (Thermo Fisher Scientific) and $10 \mathrm{mM}$ DTT. Eluted proteins were analysed by immunoblot.

Immunoblotting. Protein samples were prepared with NuPAGE LDS sample buffer (Thermo Fisher Scientific) supplemented with $10 \mathrm{mM}$ DTT. Samples were then resolved by SDS-PAGE using 4-20\% Mini-PROTEAN TGX stain-free gels (Biorad) 
and transferred onto nitrocellulose membrane using the Trans-Blot Turbo transfer system (Biorad). Membranes were blocked in 5\% (wt/vol) dried milk in TBS-T (10 $\mathrm{mM}$ Tris/ $\mathrm{HCl}, \mathrm{pH} 8,150 \mathrm{mM} \mathrm{NaCl}$ and $0.05 \%$ (vol/vol) Tween-20) for $1 \mathrm{~h}$ at RT. Membranes were incubated with primary antibody diluted in $5 \%$ (wt/vol) dried milk in TBS-T and then with the appropriate horseradish peroxidase (HRP)-conjugated secondary antibody diluted in 5\% (wt/vol) dried milk in TBS-T for $1 \mathrm{~h}$. Membranes were developed using Clarity Western ECL substrate (Biorad). Membranes were then visualized using X-ray film (Fujifilm) developed using an X-OMAT 2000 processor (KODAK) or were visualized using a ChemiDoc MP Imaging System with Image Lab 6.0 (Biorad). Secondary antibodies on membranes were inactivated by incubation with $30 \%$ hydrogen peroxide before re-probe. Primary antibodies used were: NLRP3 clone Cryo-2 at 1:1000 (AG-20B-0014, Adipogen), NLRP3 clone D4D8T at 1:1000 (15101, Cell Signaling Technology), NEK7 clone EPR4900 at 1:5000 (ab133514, Abcam), mCherry polyclonal at 1:5000 (5993, Biovision), and GAPDH polyclonal at 1:5000 (2275-PC, R\&D Systems). Secondary HRP-conjugated antibodies used were anti-rabbit IgG and anti-mouse IgG both at 1:5000 (7074, 7076, Cell Signaling Technology).

Immunoprecipitation assay. Differentiated BMM were plated at a density of $5 \times 10^{5}$ cells $/ \mathrm{ml}$ in $10 \mathrm{~cm}$ dishes in full media. The next day media were removed and replaced with Opti-MEM supplemented with CSF-1. Cells were primed with ultrapure E. coli K12 LPS (100 ng/ml) for $6 \mathrm{~h}$, or were primed for $4 \mathrm{~h}$ and stimulated with $5 \mu \mathrm{M}$ nigericin (Sigma-Aldrich) for 30 min where indicated. Media were removed and cells were washed in PBS containing MCC950 (0.01-10 $\mu \mathrm{M})$ or vehicle control. Cells were lysed in lysis buffer (50 mM Tris-HCl pH 7.4, $150 \mathrm{mM} \mathrm{NaCl}, 1 \mathrm{mM}$ ATP, 2 mM EDTA, $0.5 \%$ Igepal CA-630) containing protease inhibitors, benzonase, and MCC950 (0.01$10 \mu \mathrm{M})$ or vehicle control. Lysates were disrupted by passage through a 27-gauge needle and cleared by centrifugation at $14,000 \mathrm{G}$ for $10 \mathrm{~min}$ at $4{ }^{\circ} \mathrm{C}$. Clarified lysates were spiked with additional MCC950 $(0.01-10 \mu \mathrm{M})$ and incubated with $\alpha$-NLRP3 antibody (D4D8T) at 1:1000 and Dynabeads Protein G (Life Technologies) with gentle rotation at $4{ }^{\circ} \mathrm{C}$ overnight. The beads were washed four times with lysis buffer containing MCC950 $(0.01-10 \mu \mathrm{M})$ and immunoprecipitated samples and input $(2 \%)$ were analyzed by immunoblot.

NLRP3 inflammasome and MCC950 washout assay. Differentiated BMM were plated at a density of $1 \times 10^{6} \mathrm{cells} / \mathrm{ml}$ in full media. The next day media were removed and replaced with Opti-MEM (Life Technologies) supplemented with CSF1. Cells were primed with ultrapure E.coli K12 LPS (100 ng/ml) for $4 \mathrm{~h}$. Medium was removed and replaced with Opti-MEM containing MCC950 $(0.001-10 \mu \mathrm{M})$. For washout cells only, after 30 min the medium was removed and replaced with OptiMEM, cells were incubated for $1 \mathrm{~min}$, and medium was again removed and replaced with Opti-MEM. Cells were then stimulated with $5 \mu \mathrm{M}$ nigericin or $1.25 \mathrm{mM}$ adenosine 5'-triphosphate (ATP) (Sigma-Aldrich) for $1 \mathrm{~h}$. IL-1 $\beta$ in cell-free supernatants was analysed by ELISA (eBioscience). Cellular cytotoxicity was quantified using the Cytox96 non-radioactive cytotoxicity assay (Promega) and displayed as a percentage of total cellular LDH (100\% cell lysis control).

Recombinant NLRP3 protein expression. Human NLRP3 $\triangle L R R$ (NCBI isoform e, NP_001230062.1, residues 1-649) was cloned in PGEX-6p-1 and expressed in BL21 
competent E. coli as an N-terminal GST-fusion protein for $8 \mathrm{~h}$ at $18^{\circ} \mathrm{C}$ using $200 \mu \mathrm{M}$ Isopropyl $\beta$-D-1-thiogalactopyranoside (IPTG) (Bioline). Bacteria were then sonicated and GST-NLRP3 $\triangle$ LRR was isolated using MagneGST glutathione paramagnetic particles (Promega). GST was cleaved off by incubating the MagneGST particles with $3 \mathrm{C}$ protease for $1 \mathrm{~h}$ at RT. NLRP3 $\triangle \mathrm{LRR}$ was concentrated using Amicon Ultra Centrifugal filter units (Merck) and stored at $-80^{\circ} \mathrm{C}$. The Walker B mutant (D300A, D303A, E304A) was generated using a New England Biolabs Q5 site-directed mutagenesis kit.

Recombinant NEK7 protein expression. Human NEK7 was inserted via ligationindependent cloning into the His tag vector PMCSG7 ${ }^{32}$ and expressed in BL21(DE3) competent $E$. coli as an $\mathrm{N}$-terminal His tag-fusion protein for $15 \mathrm{~h}$ at $20^{\circ} \mathrm{C}$ using 1 mM IPTG. Bacteria were harvested, resuspended in lysis buffer (50 mM HEPES, pH $8.0,300 \mathrm{mM} \mathrm{NaCl}, 30 \mathrm{mM}$ imidazole, $1 \mathrm{mM} \mathrm{DTT}$, and $1 \mathrm{mM} \mathrm{PMSF}$ ) and disrupted by sonication. His-tag NEK7 was isolated by immobilized nickel affinity chromatography using a 5-ml HisTrap Fast Flow column (GE Healthcare). The protein was block eluted in $500 \mathrm{mM}$ imidazole and further purified using a Superdex 200 SEC column (GE Healthcare). Peak fractions were combined and flash frozen in liquid nitrogen for storage at $-80^{\circ} \mathrm{C}$.

Surface plasmon resonance (SPR) analysis of recombinant proteins. SPR for affinity and competition analysis was performed using a ForteBio Pioneer SPR system. NLRP3 $\triangle L R R$, NLRP3 $\triangle L R R$ Walker B mutant, or NEK7 were loaded onto flow cell 1 and 3 of a $\mathrm{COOH} 5$ chip and flow cell 2 was blank immobilized to enable reference subtraction in PBS. A OneStep and NextStep analysis of each of MCC950, ATP, and ADP was programmed using the Pioneer instrument software package. A maximum concentration for the analysis of $20 \mu \mathrm{M}$ was used for both OneStep and NextStep analysis. OneStep was performed with $75 \%$ loop volume and a $4 \%$ sucrose control. NextStep was performed with a 60 second injection time with each molecule and buffer as the A-component and each molecule and buffer as B components. Analysis of each cycle, OneStep and NextStep were completed separately with Qdat analysis software package. All analysis were performed on three independently prepared $\mathrm{COOH} 5$ chips with each protein loaded twice and three technical replicates per run.

\section{Statistics}

Standard deviation (S.D.) and standard error of the mean (S.E.M.) were calculated, and nonlinear regression analysis was performed using GraphPad Prism 7 software.

\section{Methods-only References}

26. Mariathasan, S., et al. Nature 430, 213-218 (2004).

27. Schroder, K., et al. P Natl Acad Sci USA 109, E944-E953 (2012).

28. Lomenick, B., Jung, G., Wohlschlegel, J.A. \& Huang, J. Curr Protoc Chem Biol 3, 163-180 (2011).

29. Pai, M.Y., et al. Methods Mol Biol 1263, 287-298 (2015).

30. Xu, C.P., et al. Chem Biol 16, 980-989 (2009).

31. Mackinnon, A.L. \& Taunton, J. Curr Protoc Chem Biol 1, 55-73 (2009).

32. Stols, L., et al. Protein Expr Purif 25, 8-15 (2002). 


\section{Supplementary Tables}

\begin{tabular}{|l|l|l|l|}
\hline $\begin{array}{l}\text { NLRP3 } \\
\text { WT }\end{array}$ & $\mathbf{k}_{\mathbf{a}}\left(\mathbf{M}^{-1} \mathbf{s}^{-1}\right)$ & $\mathbf{k}_{\mathbf{d}}\left(\mathbf{s}^{-1}\right)$ & $\mathbf{K}_{\mathbf{D}}$ \\
\hline MCC950 & $1.10 \times 10^{6} \pm 0.2$ & $0.247 \pm 0.003$ & $224 \mathrm{nM} \pm 39.6$ \\
\hline ATP & $1.18 \times 10^{7} \pm 0.02$ & $24.15 \pm 0.02$ & $2.05 \mu \mathrm{M} \pm 0.03$ \\
\hline MCC950 + ATP & $1.39 \times 10^{5} \pm 0.01$ & $1.47 \times 10^{-3} \pm 0.02$ & $10.6 \mathrm{nM} \pm 0.2$ \\
\hline ADP & $1.11 \times 10^{3} \pm 0.3$ & $0.48 \pm 0.01$ & $432 \mu \mathrm{M} \pm 107$ \\
\hline MCC950 + ADP & $100 \pm 60$ & $0.295 \pm 0.02$ & $2.95 \mathrm{mM} \pm 1.1$ \\
\hline $\begin{array}{l}\text { NLRP3 } \\
\text { Walker B mutant }\end{array}$ & $\mathbf{k}_{\mathbf{a}}\left(\mathbf{M}^{-1} \mathbf{s}^{-1}\right)$ & $\mathbf{k}_{\mathbf{d}}\left(\mathbf{s}^{-1}\right)$ & $\mathbf{K}_{\mathbf{D}}$ \\
\hline MCC950 & No Interaction & & \\
\hline ATP & $3.59 \times 10^{3} \pm 0.8$ & $0.90 \pm 0.04$ & $251 \mu \mathrm{M} \pm 51.5$ \\
\hline NEK7 & $\mathbf{k}_{\mathbf{a}}\left(\mathbf{M}^{-1} \mathbf{s}^{-1}\right)$ & $\mathbf{k}_{\mathbf{d}}\left(\mathbf{s}^{-1}\right)$ & $\mathbf{K}_{\mathbf{D}}$ \\
\hline MCC950 & No Interaction & & \\
\hline ATP & $4.7 \times 10^{3} \pm 0.8$ & $0.321 \pm 0.009$ & $70 \mu \mathrm{M} \pm 10$ \\
\hline
\end{tabular}

Supplementary Table 1. Surface plasmon resonance analysis of NLRP3ALRR, NLRP3ALRR-Walker B, and NEK7 recombinant proteins with MCC950, ATP, and ADP. Values for on rate $\left(\mathrm{K}_{\mathrm{a}}\right)$, off rate $\left(\mathrm{K}_{\mathrm{d}}\right)$ and dissociation constant $\left(\mathrm{K}_{\mathrm{D}}\right)$ are shown \pm S.D. $\mathrm{N}=3$ independent experiments, where protein was loaded twice and three technical replicates per run were performed. 


\section{Supplementary Figures}

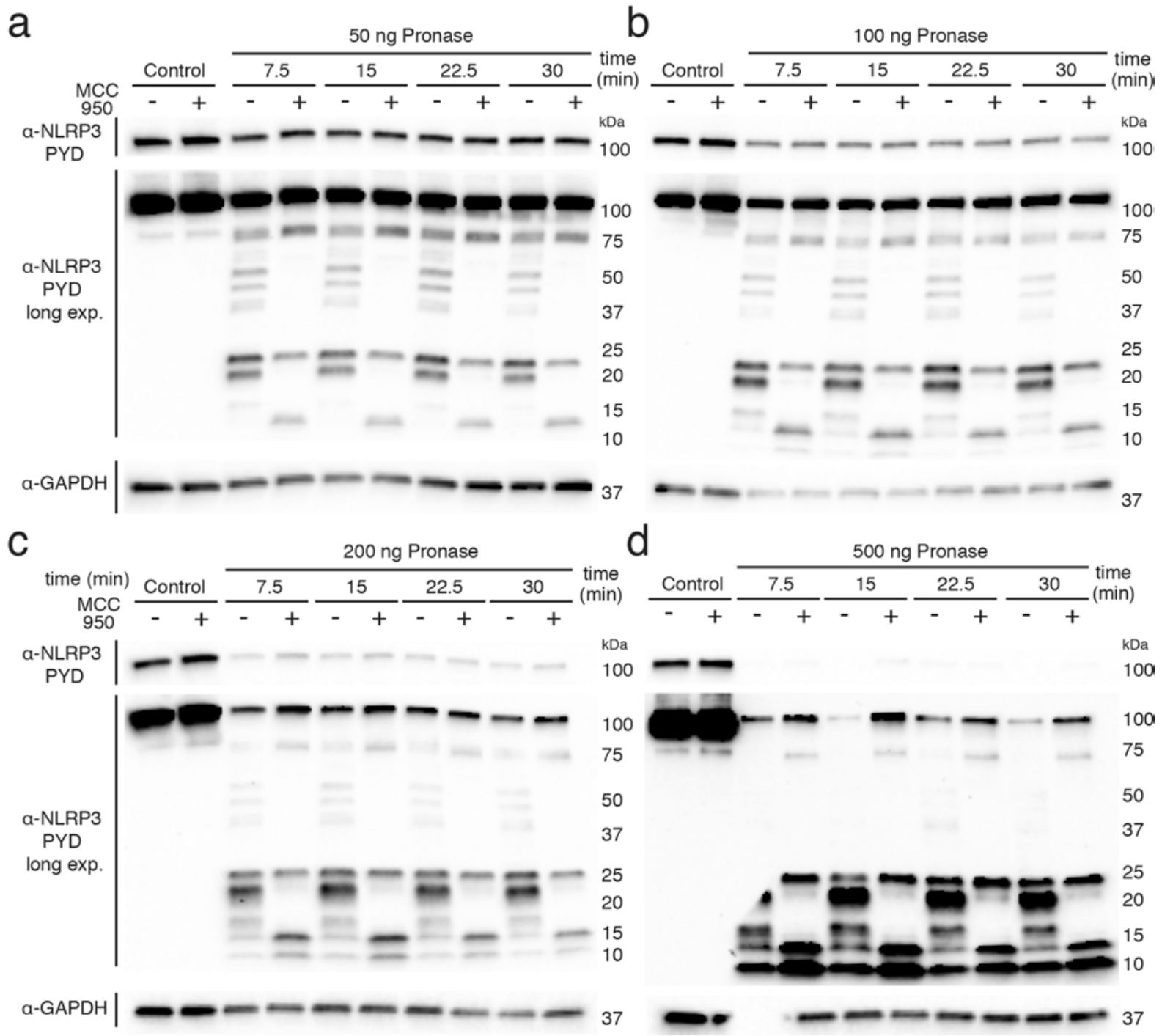

Supplementary Figure 1. Optimisation of DARTS assays. (a-d) LPS-primed BMM were treated with MCC950 $(10 \mu \mathrm{M})$ or vehicle for $1 \mathrm{~h}$, and cells were lysed in buffer +/- MCC950. DARTS assay was performed with pronase at (a) $50 \mathrm{ng}$, (b) $100 \mathrm{ng}$, (c) $200 \mathrm{ng}$, or (d) $500 \mathrm{ng}$ per $\mu \mathrm{g}$ of protein in the lysate samples. Samples were incubated for 7.5-30 min as indicated. Lysates were analysed by immunoblot using antibodies for NLRP3 PYD (cryo-2) and GAPDH. Data are representative of $\mathrm{N}=2$ independent experiments. Uncropped Western blots are shown in Supplementary Figure 12. 
a
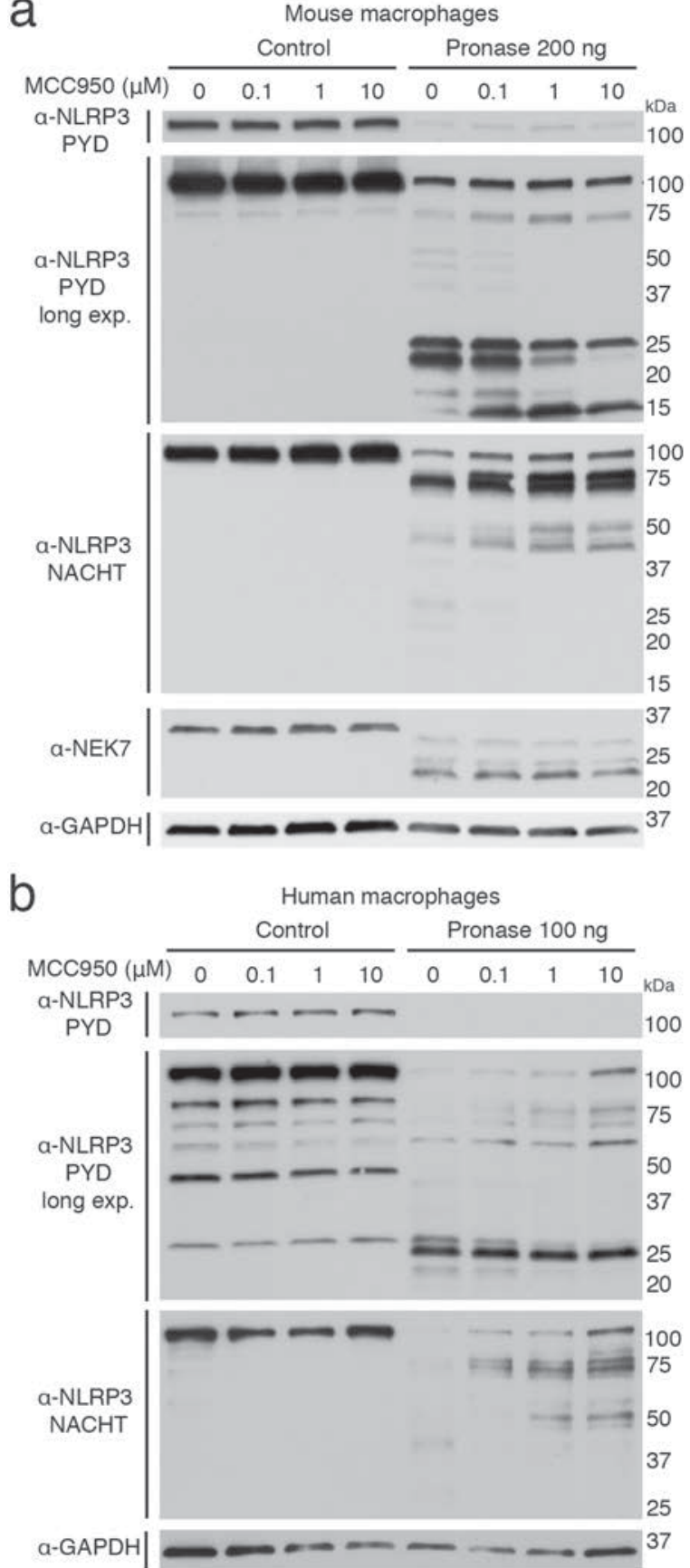

C

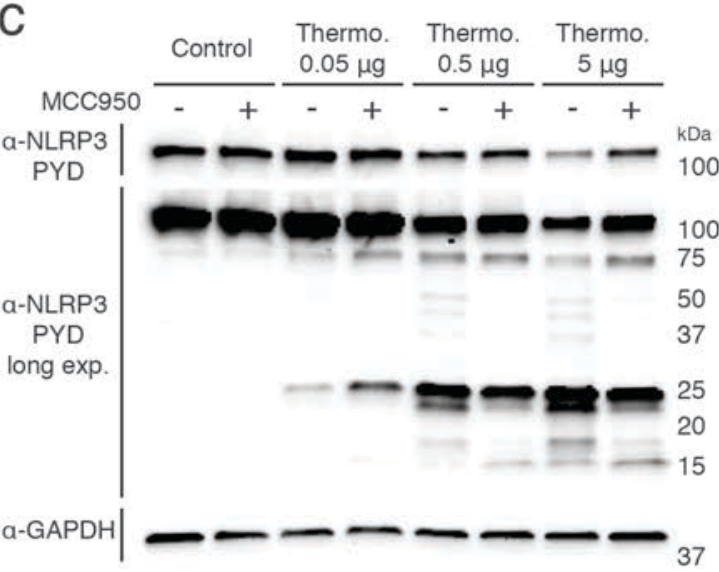

d

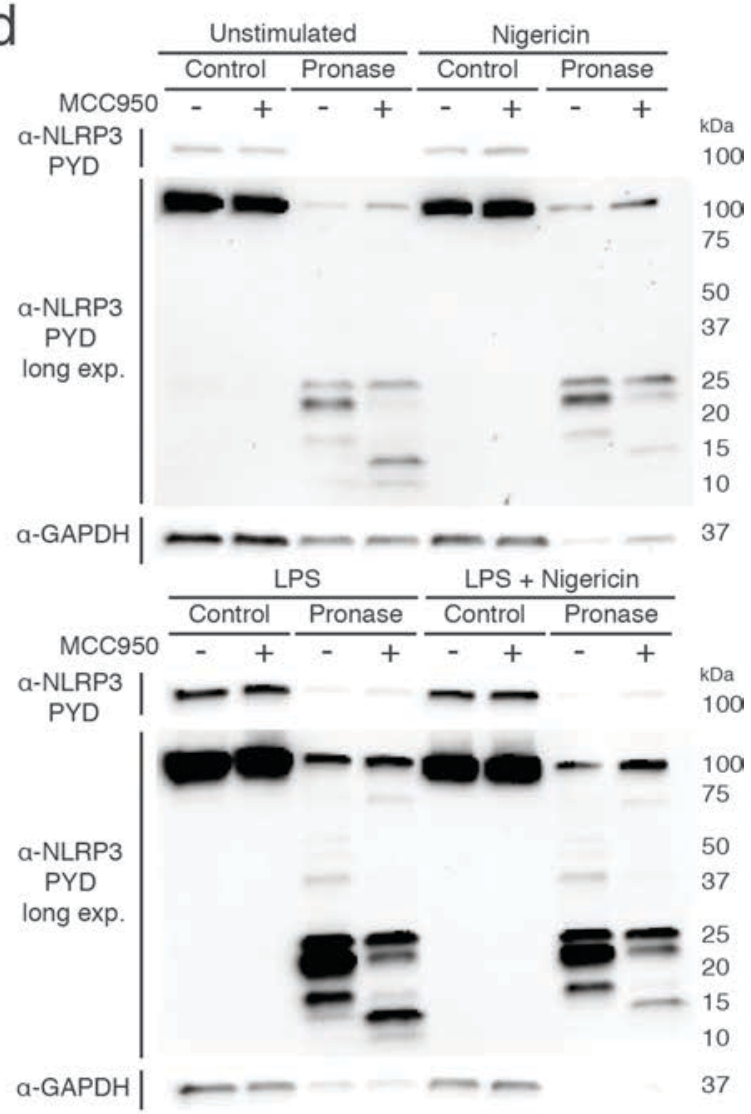

Supplementary Figure 2. MCC950 blocks NLRP3 degradation in different cell types, with an alternative protease, and under different activation conditions. LPS-primed BMM (a) or human monocyte-derived macrophages (b) were treated with MCC950 (0.1-10 $\mu \mathrm{M})$ or vehicle for $1 \mathrm{~h}$, and cells were lysed in buffer +/- MCC950 (0.1-10 $\mu \mathrm{M})$. DARTS assay was performed with (a) $200 \mathrm{ng}$ or (b) $100 \mathrm{ng}$ pronase per $\mu \mathrm{g}$ protein. (c) LPS-primed BMM were treated with MCC950 $(10 \mu \mathrm{M})$, and DARTS assays performed with thermolysin at 0.05-5 $\mu \mathrm{g}$ per $\mu \mathrm{g}$ of protein. (d) Asc ${ }^{-1-B M M}$ were unstimulated or treated with LPS (4 h) and/or nigericin (30 min), cells were lysed in buffer $+/$ - MCC950 (10 $\mu \mathrm{M})$. DARTS assay was performed with $100 \mathrm{ng}$ pronase per $\mu \mathrm{g}$ of protein) (a-d) Lysates were analysed by immunoblot using antibodies for NLRP3 PYD (cryo-2), NLRP3 NACHT (D4D8T), NEK7 and GAPDH. Data are representative of (a) $\mathrm{N}=5$, (b,d) $\mathrm{N}=3$, or (c) $\mathrm{N}=2$ independent experiments. Uncropped Western blots are shown in Supplementary Figure 13. 


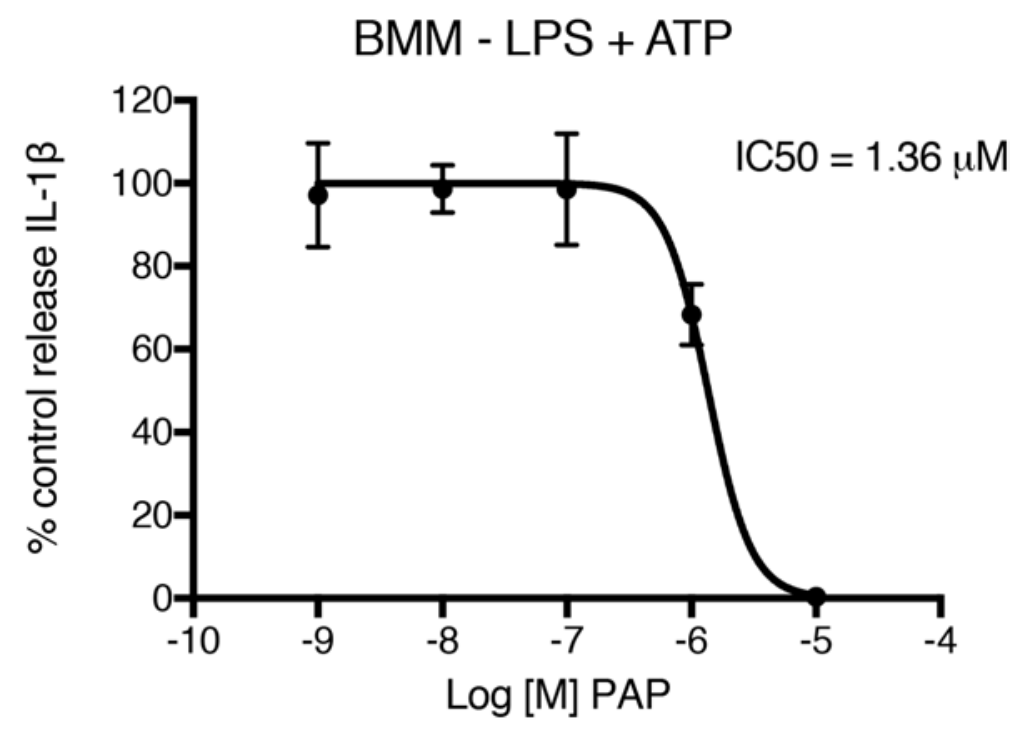

Supplementary Figure 3. The photoaffinity probe inhibits NLRP3 function. IL-1 $\beta$ secretion as measured by ELISA, from LPS-primed BMM treated with the photoaffinity probe (PAP) $(0.001-10 \mu \mathrm{M})$ in the final $30 \mathrm{~min}$ of priming and then stimulated with ATP for $1 \mathrm{~h}$. Cytokine level is normalized to that of vehicle-treated control cells. Data are mean of $\mathrm{N}=3$ independent experiments, each experiment was performed in technical triplicate, error bars show \pm SEM. Nonlinear regression analysis was performed, and the curve of Log [M] PAP versus the normalized response (variable slope) is presented. 

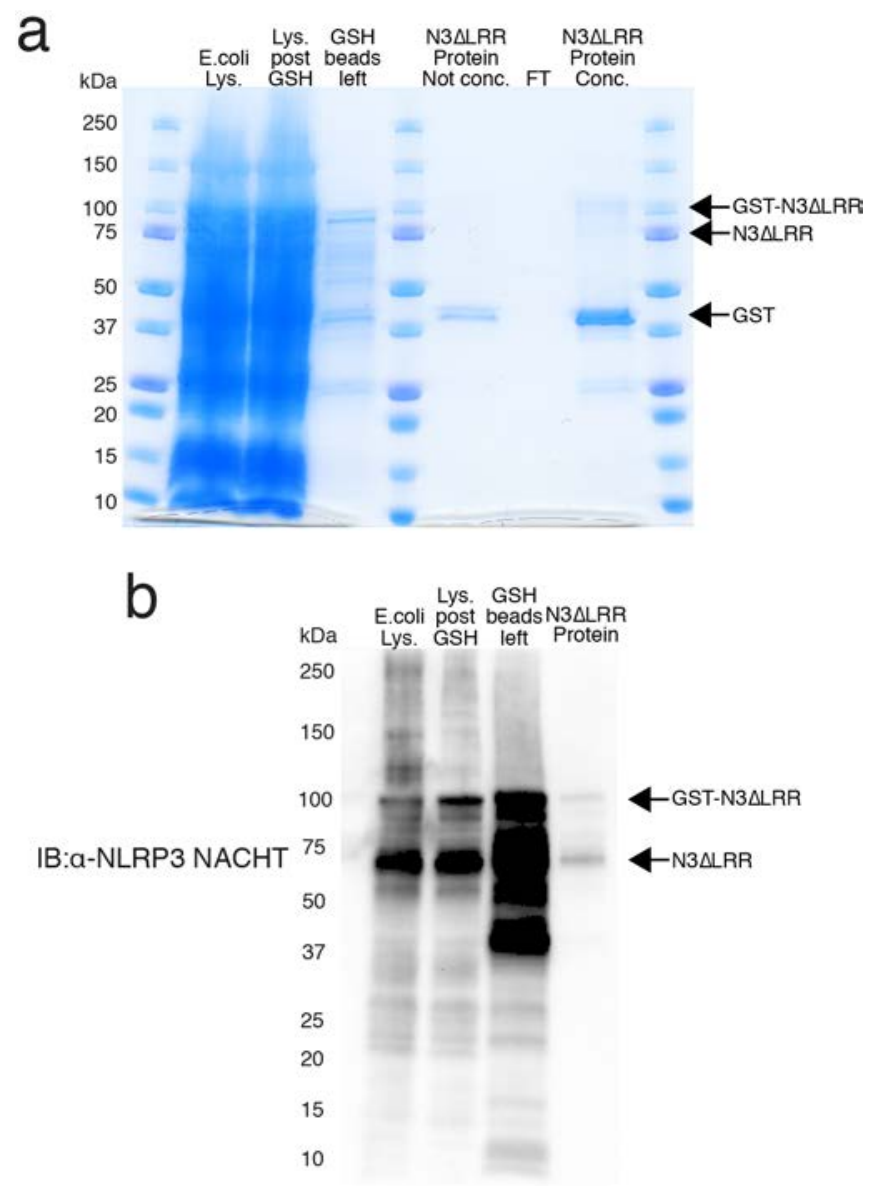

Supplementary Figure 4. Expression and purification of NLRP3 $\Delta$ LRR. SDSPAGE gel of purified human NLRP3 $\triangle$ LRR protein (a) stained with GelCode Blue reagent, or (b) subject to immunoblot analysis using an antibody to NLRP3 NACHT (D4D8T). Data are representative of $\mathrm{N}=2$ independent experiments. 


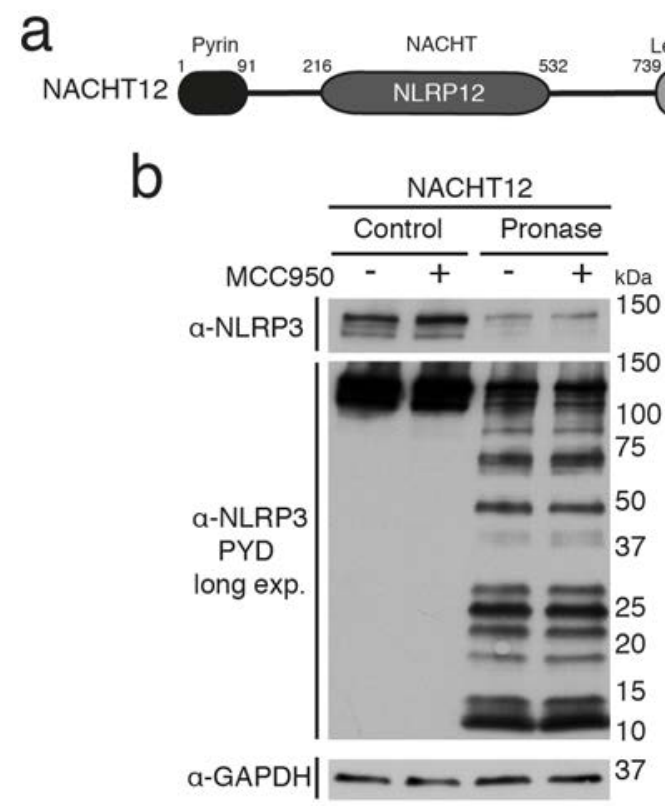

Supplementary Figure 5. MCC950 does not block degradation of a NACHT12 chimera. (a) Schematic diagram of the 'NACHT12' protein chimera used in (b), where the NACHT domain of NLRP3 is swapped for that of NLRP12. (b) HEK293T cells transfected with mCherry-tagged NLRP3-NLRP12 NACHT chimera, were lysed in buffer $+/-$ MCC950 $(10 \mu \mathrm{M})$. DARTS assays were performed with pronase $(200 \mathrm{ng} / \mu \mathrm{g}$ of protein) and lysates were analysed by immunoblot using antibodies for NLRP3 PYD and GAPDH. Data are representative $\mathrm{N}=3$ independent experiments. Uncropped Western blots are shown in Supplementary Figure 14. 


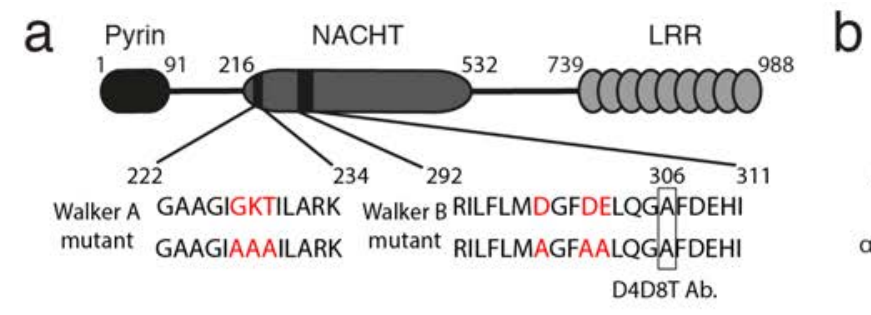

C

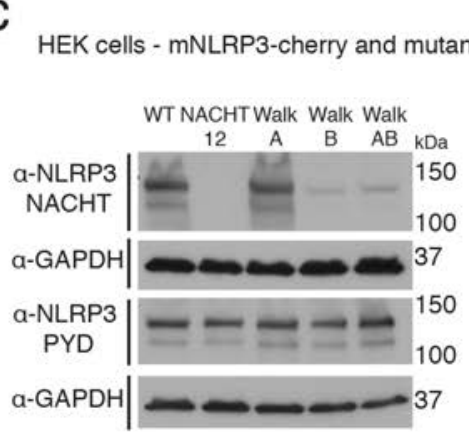

b

d
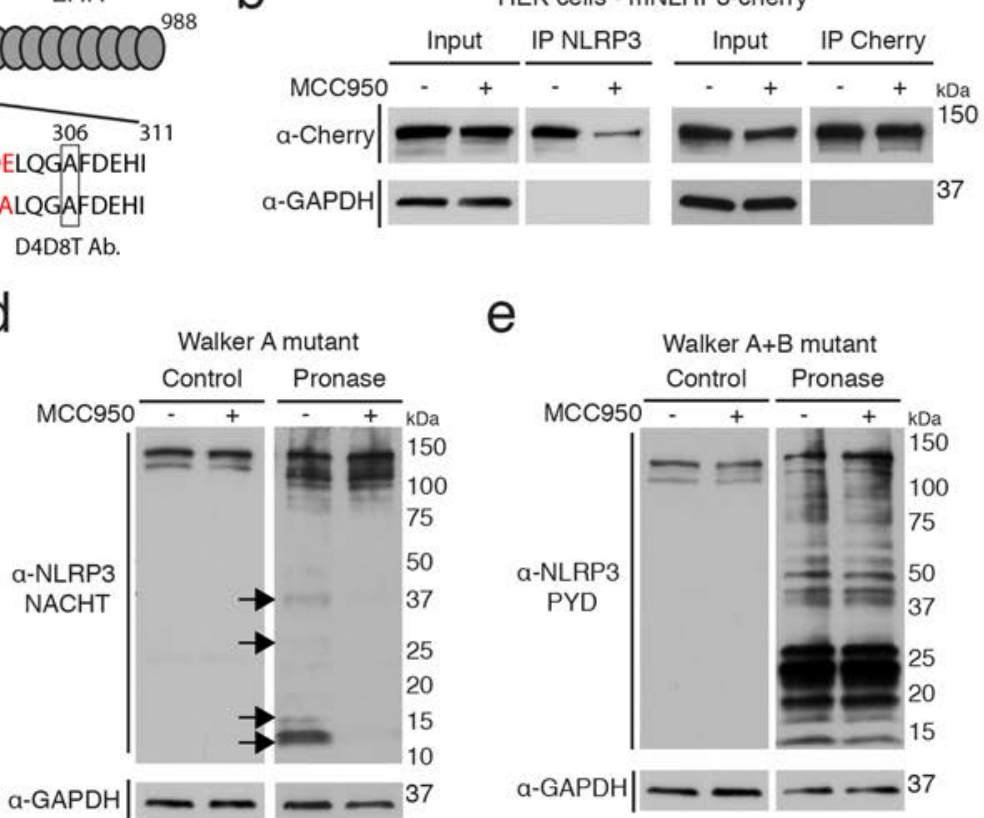

e

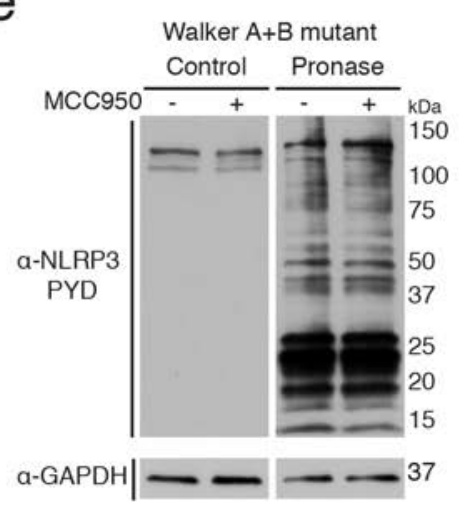

Supplementary Figure 6. MCC950 specifically blocks NLRP3 interaction with the D4D8T antibody that recognises the NLRP3 walker B motif, and limits degradation of the NLRP3 Walker A mutant but not the Walker $B$ mutant. (a) Schematic diagram of the NLRP3 NACHT domain showing the location of Ala306, the Walker A and Walker B motifs (in red) and the mutants used in (d-e). (b) HEK293T cells were transfected with mCherry-tagged WT NLRP3 for $24 \mathrm{~h}$. Cells were lysed in buffer +/- MCC950 $(10 \mu \mathrm{M})$ and NLRP3 was immunoprecipitated with the NLRP3 NACHT D4D8T antibody or an anti-mCherry antibody. NLRP3 levels in the input and IP samples were analysed by immunoblot using antibodies for mCherry and GAPDH. (c) HEK293T cells were transfected with mCherry-tagged WT NLRP3, Walker A mutant, Walker B mutant, Walker A+B double mutant and NLRP3-NLRP12 NACHT chimera (NACHT12) for $24 \mathrm{~h}$. (d-e) HEK293T cells transfected with mCherry-tagged NLRP3 Walker A mutant (d), or Walker A+B double mutant (e) for $24 \mathrm{~h}$. Cells were lysed in buffer $+/$ - MCC950 (10 $\mu \mathrm{M})$. DARTS assays were performed with pronase (200 ng/ $\mu \mathrm{g}$ of protein). (c-d) Cell lysates were analysed by immunoblot using antibodies for NLRP3 PYD (cryo-2), NLRP3 NACHT (D4D8T), and GAPDH. (d) Arrows indicate changes in degradation observed with MCC950 treatment. Data are representative of (b) $\mathrm{N}=4$, (c) $\mathrm{N}=2$, and (d,e) $\mathrm{N}=3$ independent experiments. Uncropped Western blots are shown in Supplementary Figure 15. 

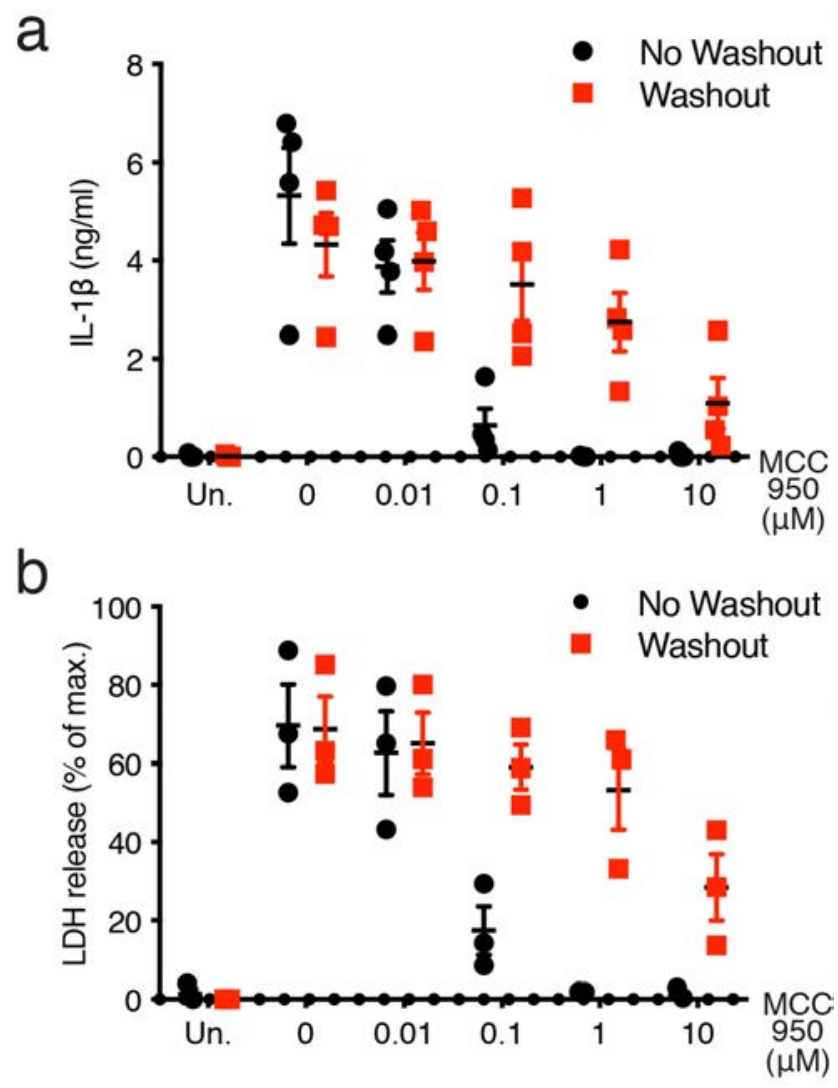

Supplementary Figure 7. The NLRP3-inhibitory function of MCC950 is reversible. LPS-primed BMM were treated with MCC950 $(0.01-10 \mu \mathrm{M})$ and stimulated with nigericin for $1 \mathrm{~h}$. MCC950 was left on cells or washed out for $1 \mathrm{~min}$ prior to nigericin treatment. (a) IL-1 $\beta$ secretion measured by ELISA. (b) Cell death measured by LDH release assay. (a) $\mathrm{N}=4$ or (b) $\mathrm{N}=3$ independent experiments, in which each experiment was performed in technical triplicate. Bars show the mean \pm S.E.M of the independent experiments. 

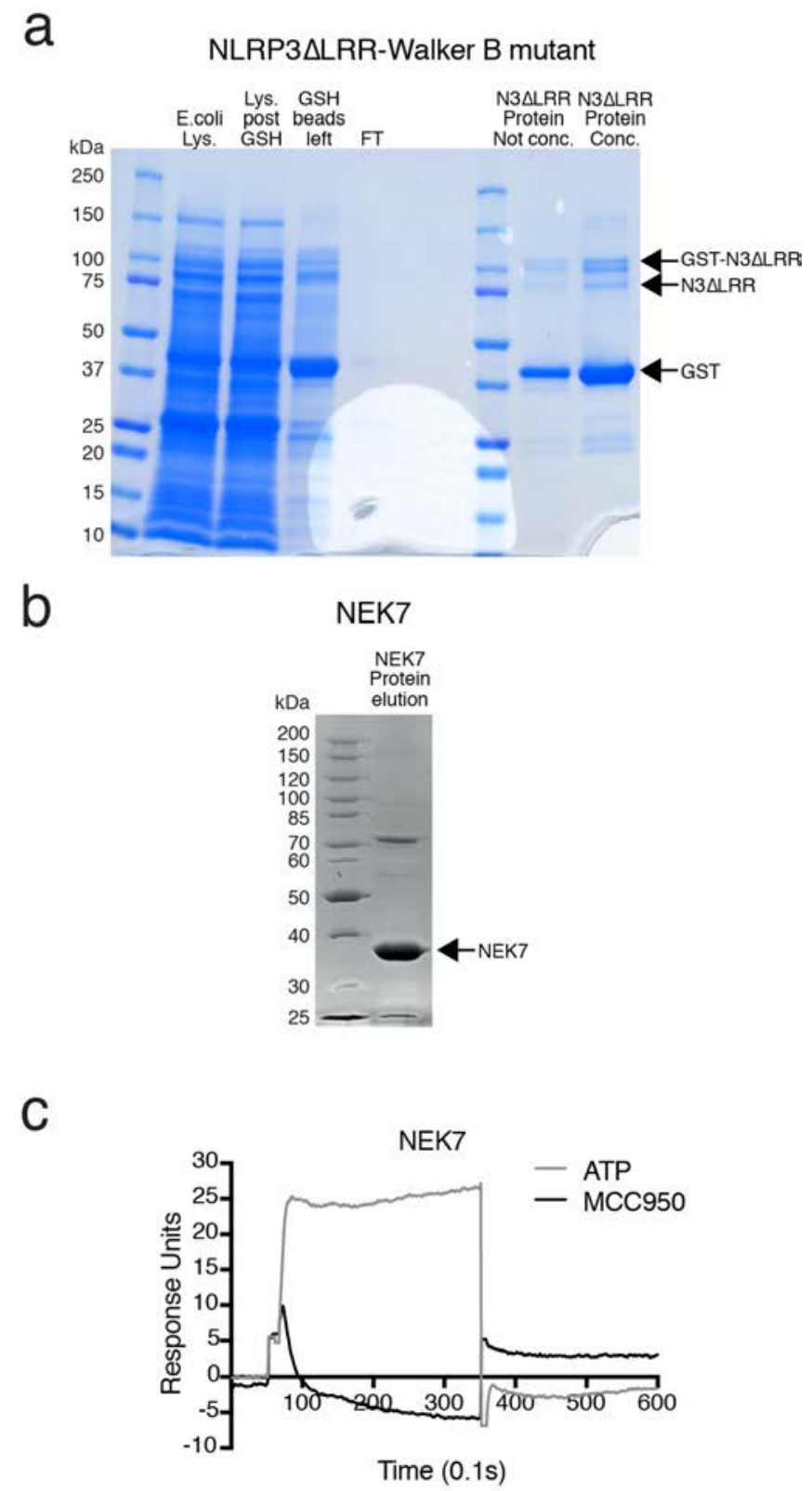

Supplementary Figure 8. Expression and purification of NLRP3 $\triangle$ LRR Walker B mutant and NEK7, and SPR analysis of NEK7. (a) SDS-PAGE analysis of human NLRP3 $\triangle$ LRR Walker B mutant protein stained with GelCode Blue reagent. (b) SDSPAGE analysis of human NEK7 protein stained with Coomassie. $(\mathbf{a}, \mathbf{b})$ These experiments were performed once. (c) Surface plasmon resonance analysis of MCC950 and ATP interaction with NEK7. A representative sensorgram is shown from $\mathrm{N}=3$ independent experiments, where protein was loaded twice and three technical replicates per run were performed. 
Figure 1a - NLRP3 cryo2

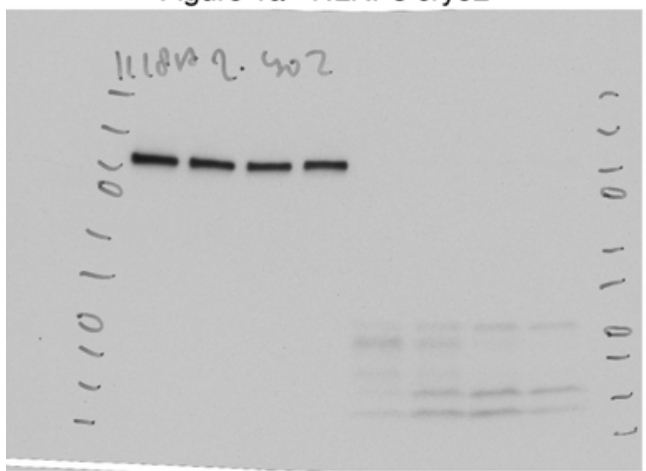

Figure 1a - NLRP3 cryo2 long exp.

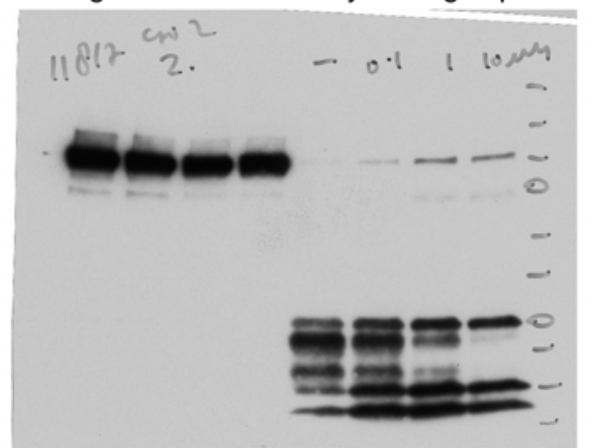

Figure 1a - NLRP3 D4D8T

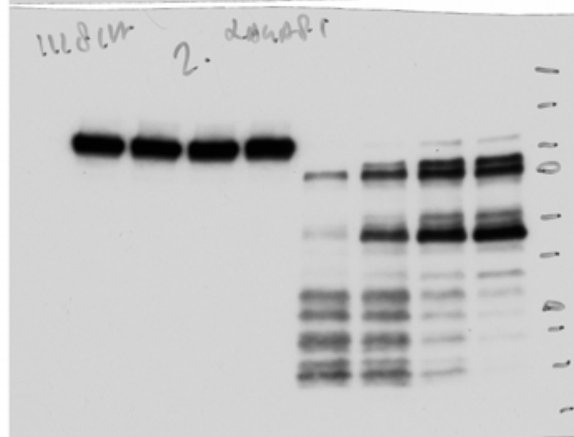

Figure 1a - NEK7

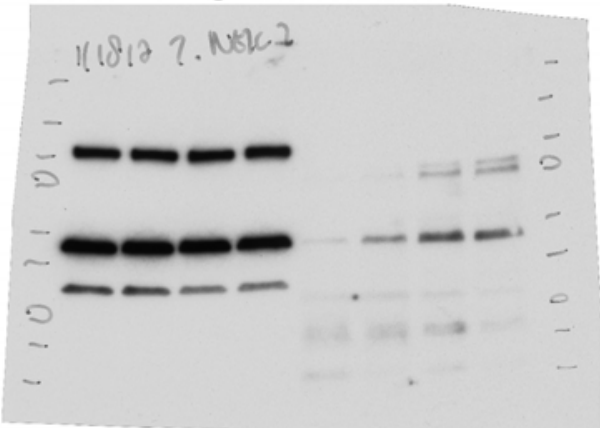

Figure 1a - GAPDH

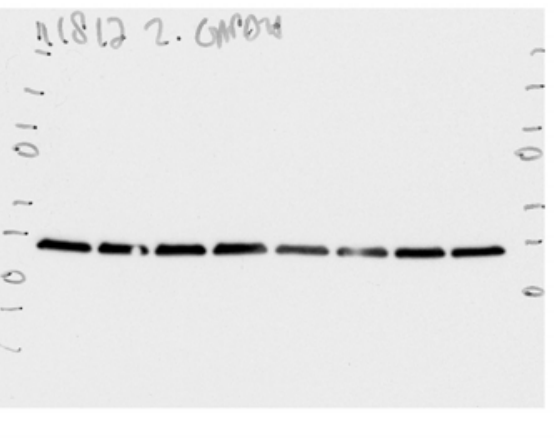

Figure 1b - NLRP3 D4D8T

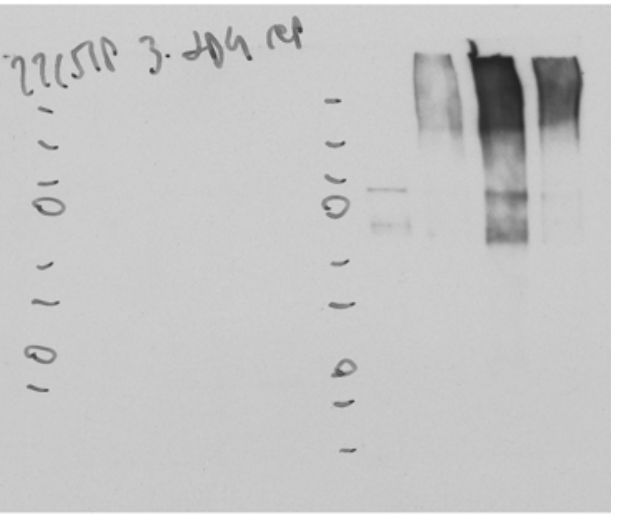

Figure 1c - NLRP3 cryo2

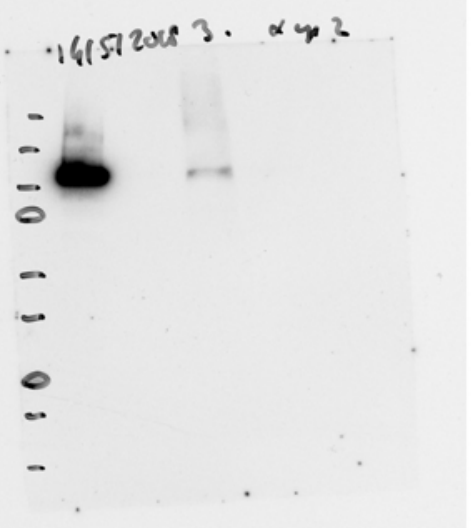

Supplementary Figure 9. Uncropped Western blots related to Figure 1. 
Figure 2b - NLRP3 D4D8T

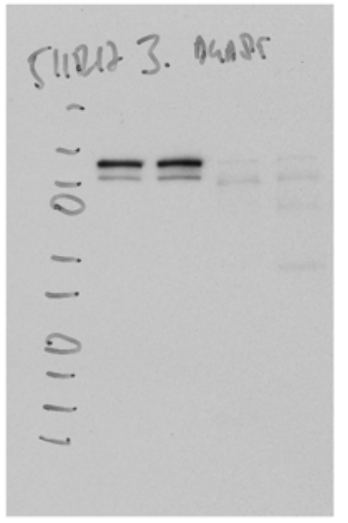

Figure 2c - NLRP3 cryo2

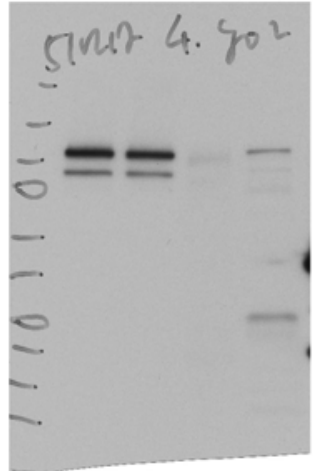

Figure 2b - NLRP3 cryo2 long exp.

Figure 2b - NLRP3 D4D8T long exp.

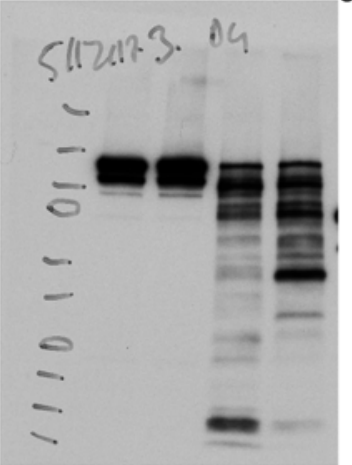

Figure 2b - GAPDH

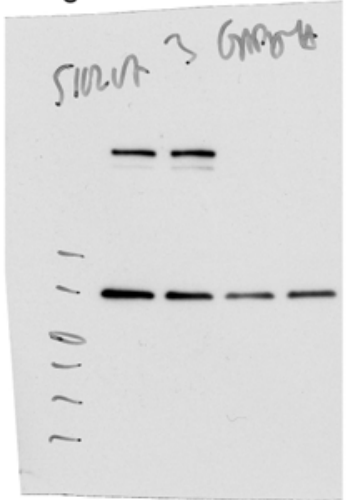

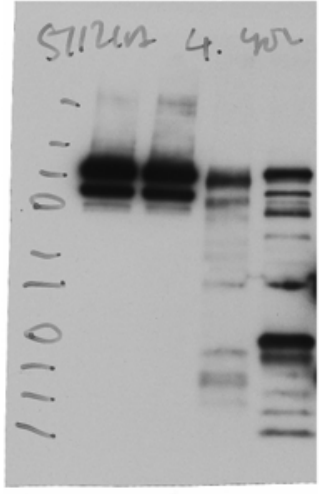

Figure 2c - GAPDH

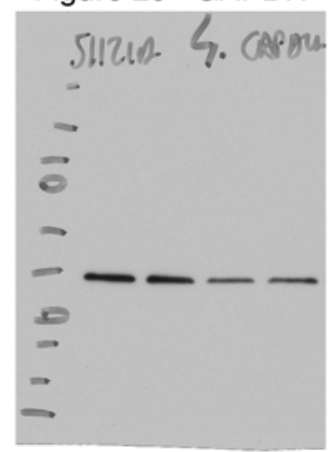

Figure 2d - NLRP3 cryo2

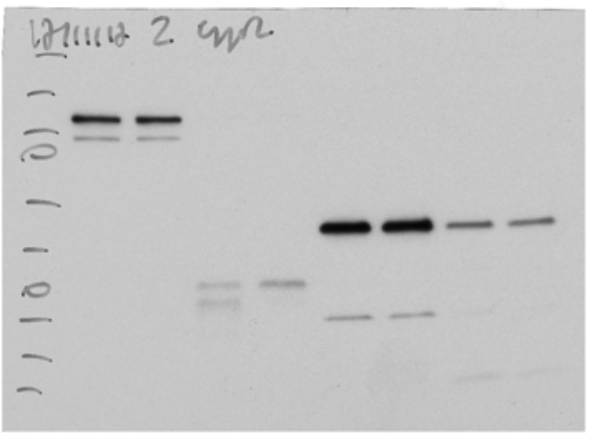

Figure 2d - NLRP3 cryo2 long exp.

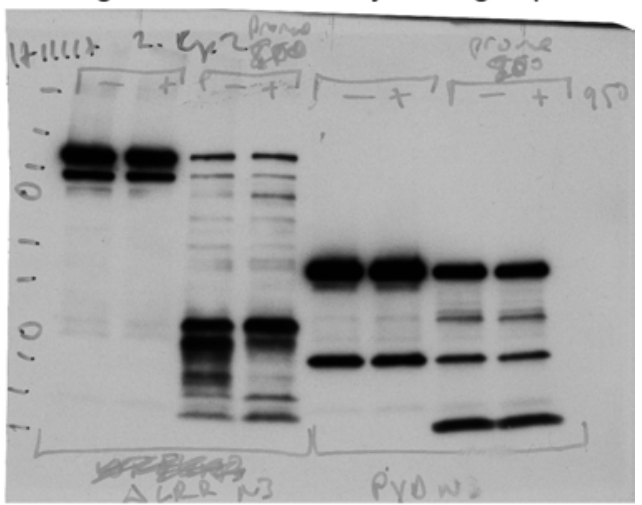

Figure 2d- GAPDH

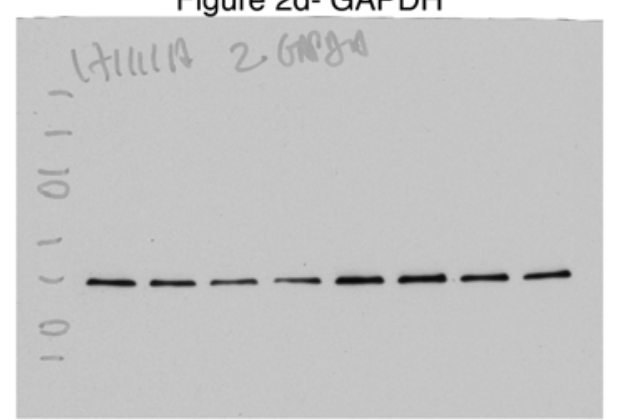

Supplementary Figure 10. Uncropped Western blots related to Figure 2. 
Figure 3a - NLRP3 cryo2

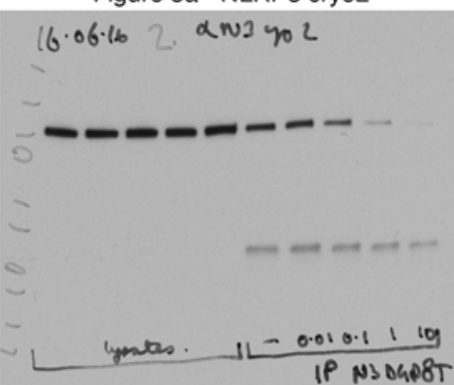

Figure 3b - IP - NLRP3 cryo2

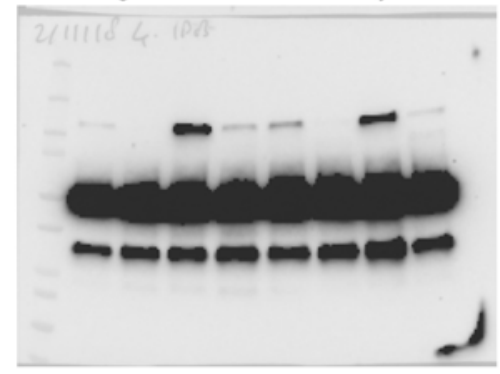

Figure 3c - control - NLRP3 D4D8T

36.46 2.04085

$\sim-$

$\sim$

$\sim$

$-$

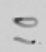

$\sim$

Figure 3d - control - NLRP3 cryo2

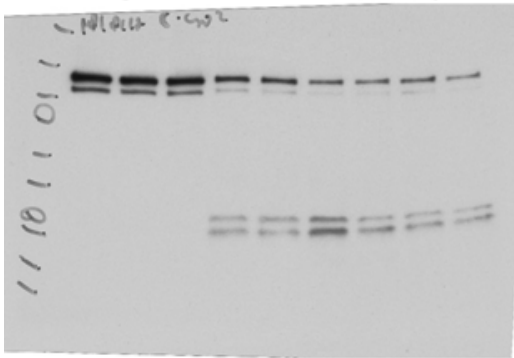

Supplementary Figure 11. Uncropped Western blots related to Figure 3.
Figure $3 a$ - GAPDH

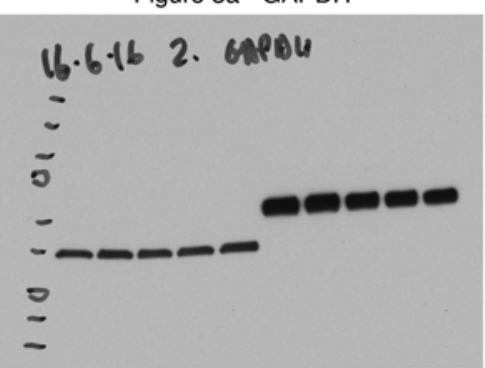

Figure $3 b$ - input - NLRP3 cryo2

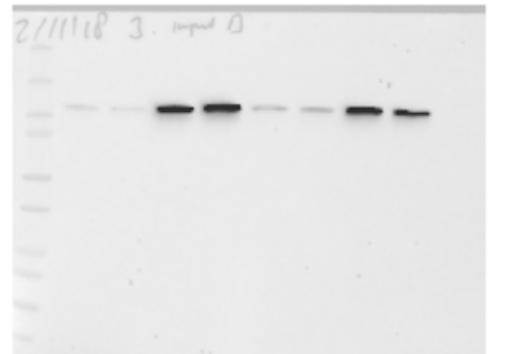

Figure 3c - pronase - NLRP3 D4D8T 3/4. 2.04005

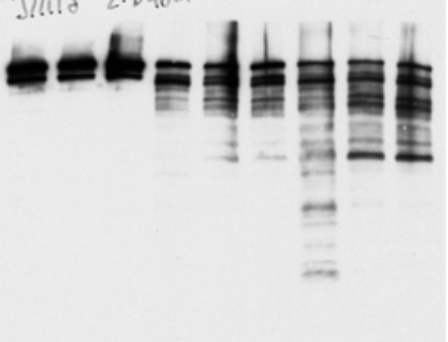

Figure 3d - pronase - NLRP3 cryo2

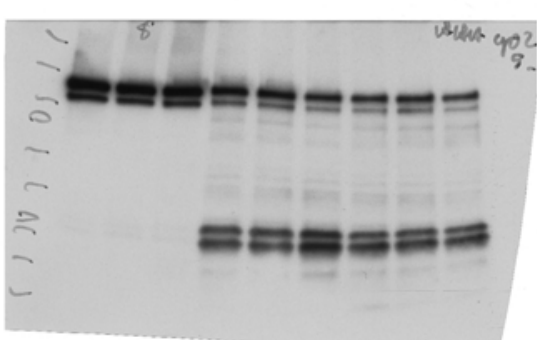

Figure $3 b$ - input - GAPDH

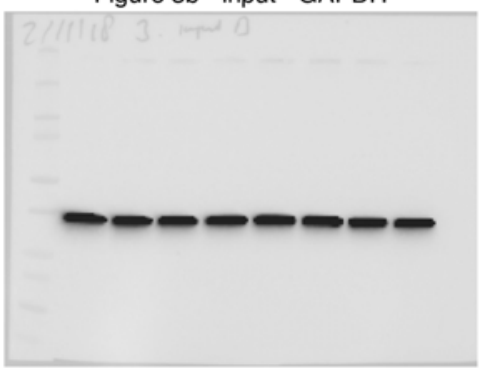

Figure 3c - GAPDH

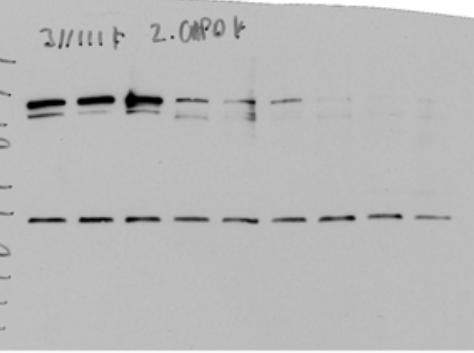

Figure 3d - GAPDH

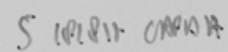

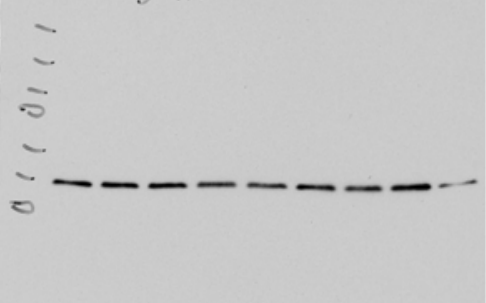




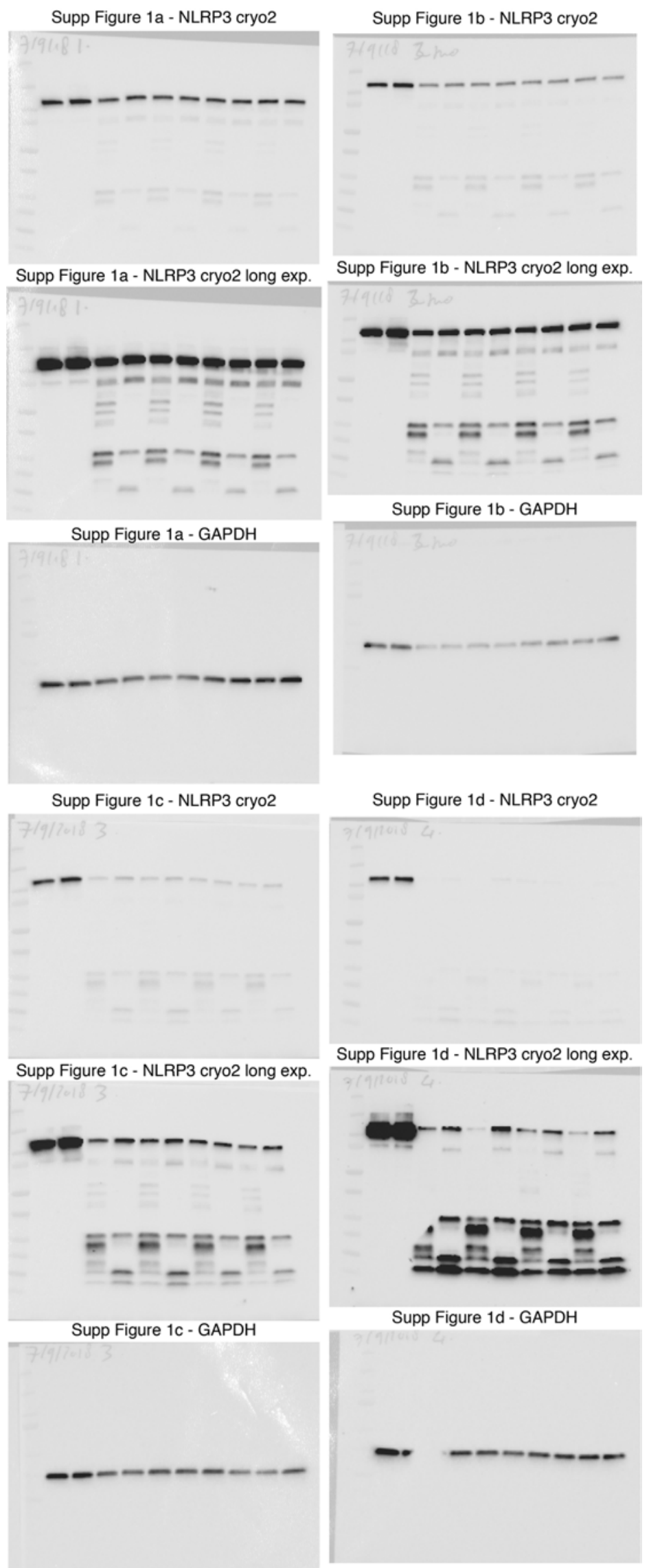

Supplementary Figure 12. Uncropped Western blots related to Supplementary Figure 1. 

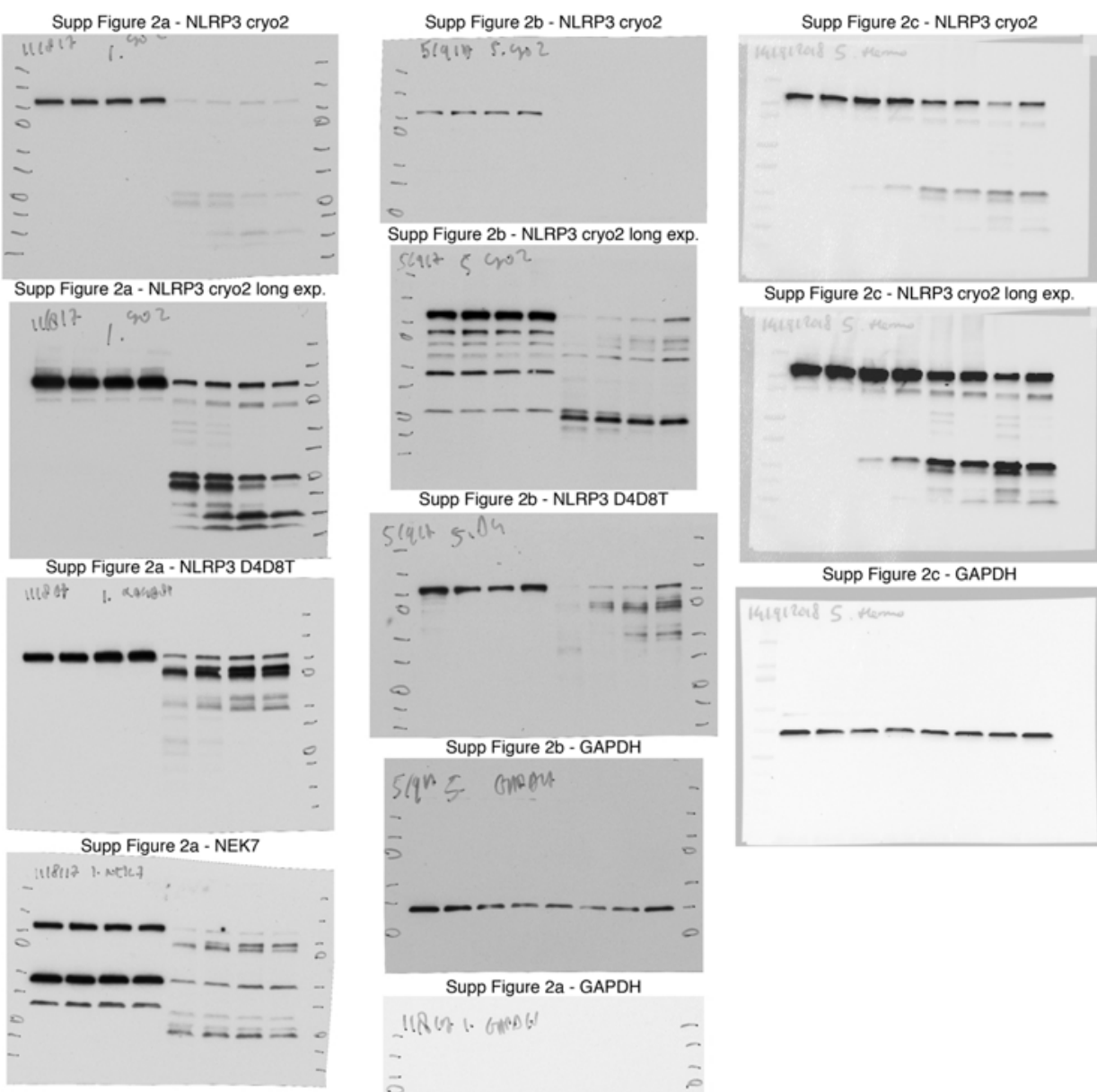

Supp Figure $2 b$ - NLRP3 cryo2 long exp. SCat? S G OO?

Supp Figure 2c - NLRP3 cryo2 long exp.

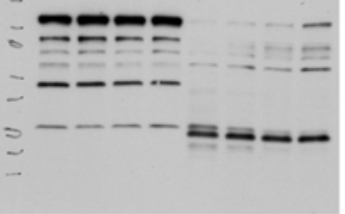

Supp Figure 2b - NLRP3 D4D8T

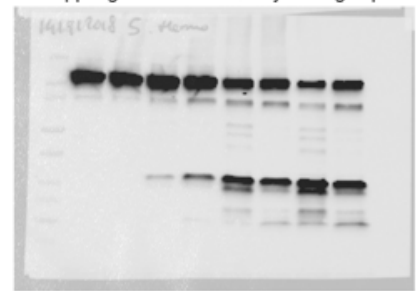

Supp Figure 2c - GAPDH

Supp Figure 2d - NLRP3 cryo2 - top

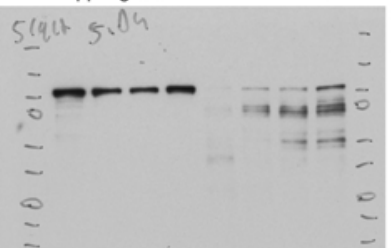

Supp Figure 2b - GAPDH

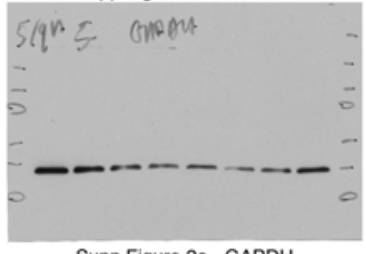

Supp Figure 2a - GAPDH

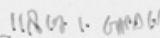

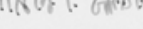

$\overrightarrow{0}$
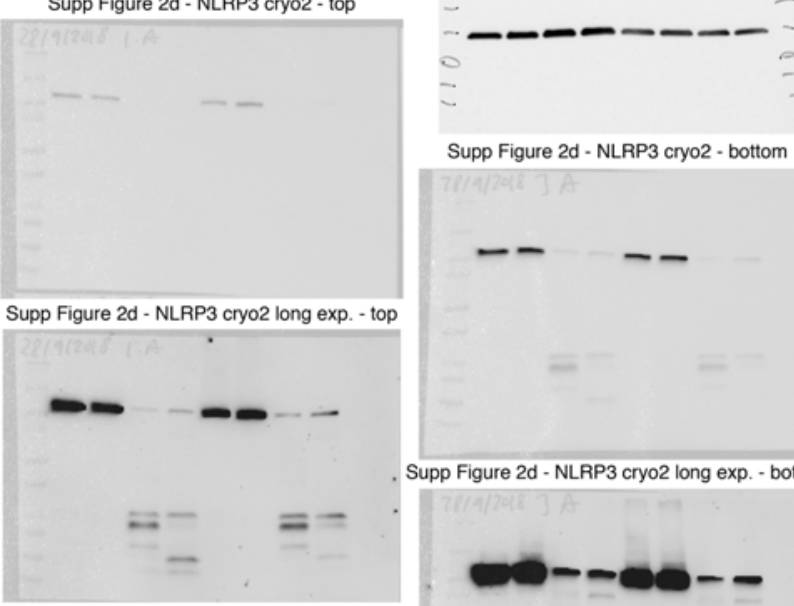

Supp Figure 2d - NLRP3 cryo2 - bottom

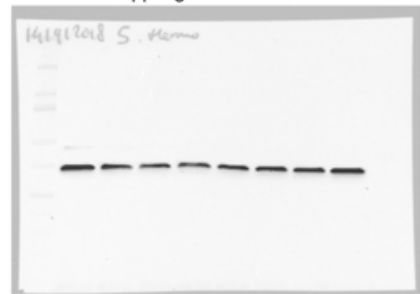

Supp Figure 2d - GAPDH - top
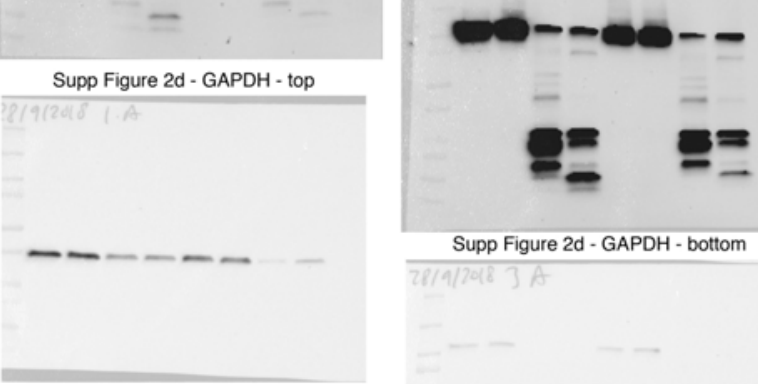

Supp Figure 2d - GAPDH - bottom

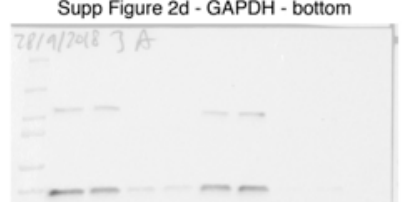

Supplementary Figure 13. Uncropped Western blots related to Supplementary Figure 2. 
Supp Figure 5b - NLRP3 cryo2

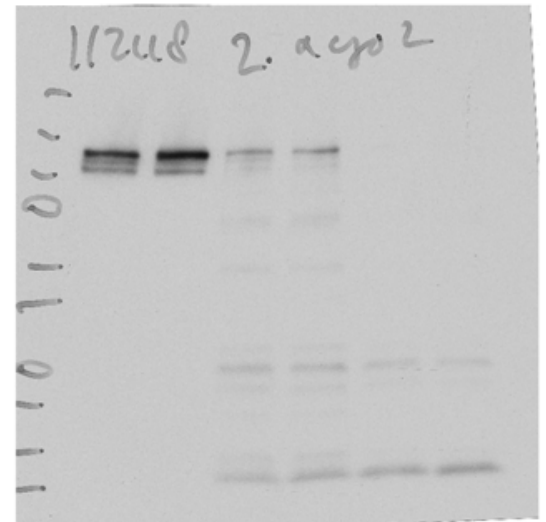

Supp Figure 5b - NLRP3 cryo2 long exp.

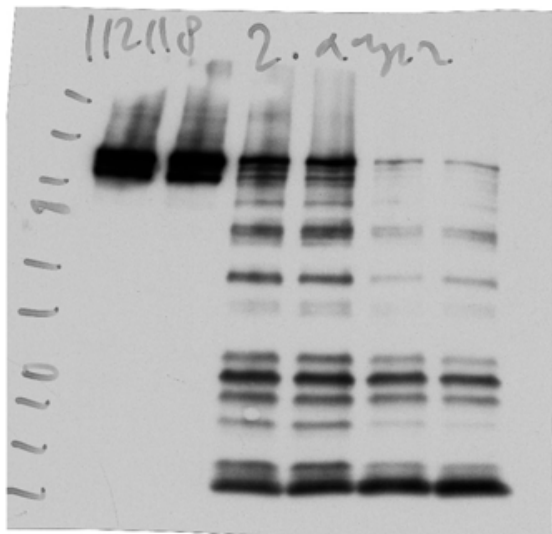

Supp Figure 5b - GAPDH

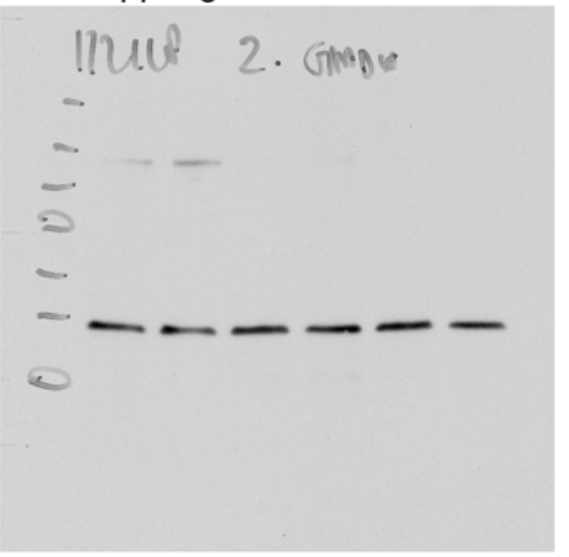

Supplementary Figure 14. Uncropped Western blots related to Supplementary Figure 5. 
Supp Figure 6b - cherry - input and IP NLRP3 Supp Figure 6c - D4D8T

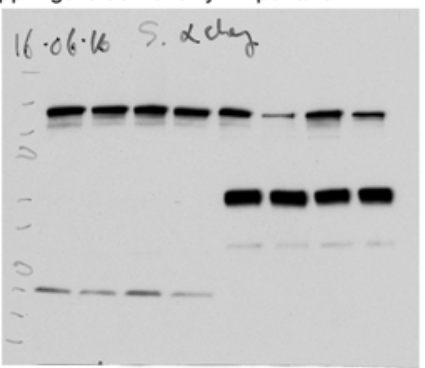

Supp Figure 6b - GAPDH - input and IP NLRP3

Supp Figure 6c - GAPDH - top

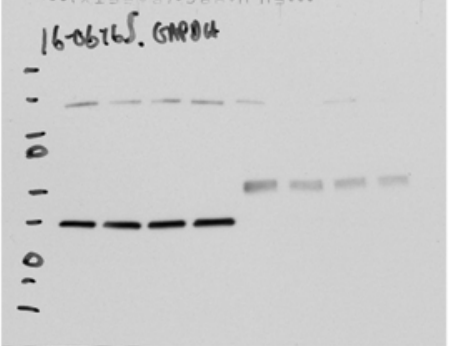

Supp Figure $6 \mathrm{~b}$ - cherry - input and IP cherry $1606.68 \mathrm{chy}$
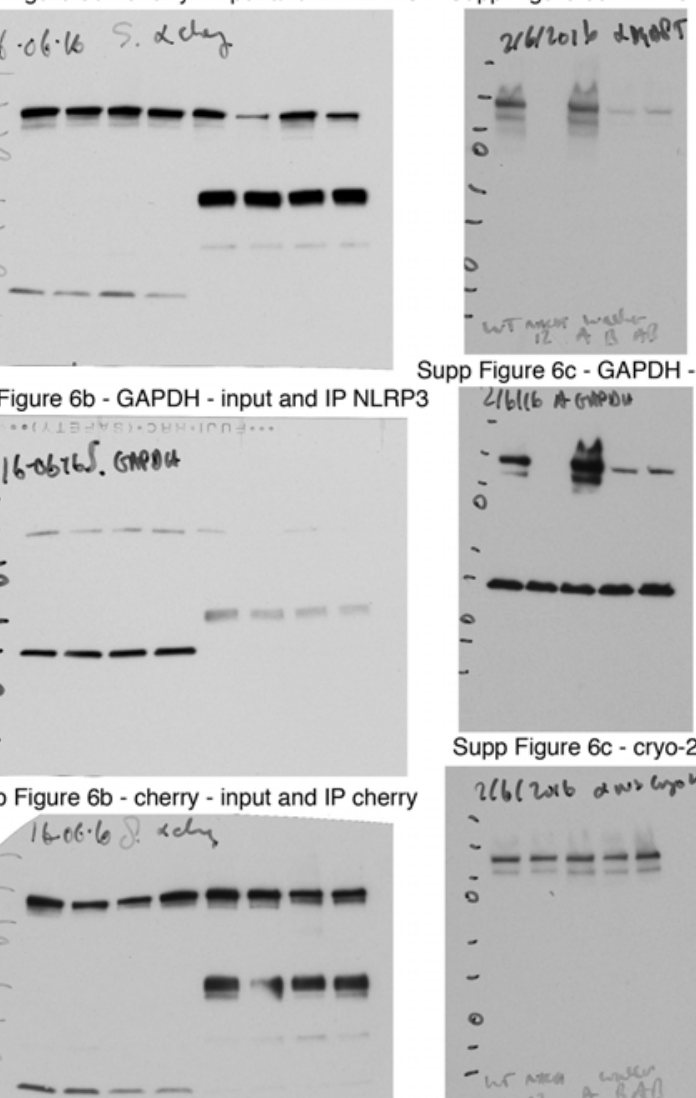

Supp Figure 6d - D4D8T - pronase

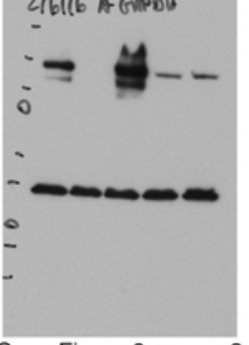

Supp Figure 6c - cryo-2

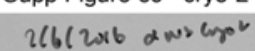

$\checkmark$

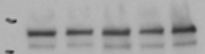

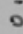

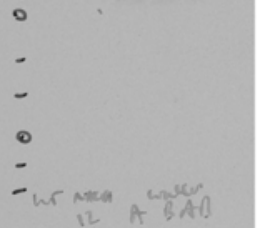

Supp Figure 6c - GAPDH - bottom

Supp Figure 6b - GAPDH - input and IP cherry
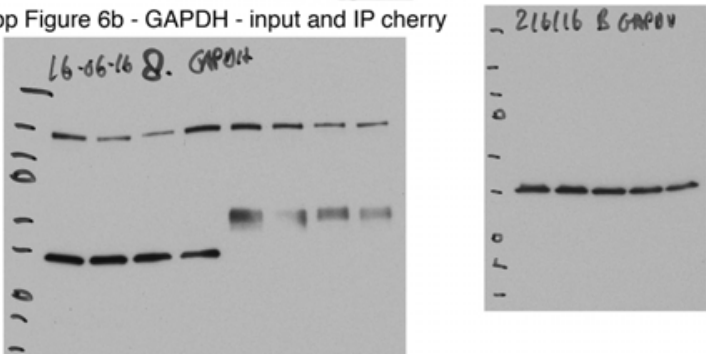

Supplementary Figure 15. Uncropped Western blots related to Supplementary Figure 6.
Supp Figure 6d - D4D8T - control

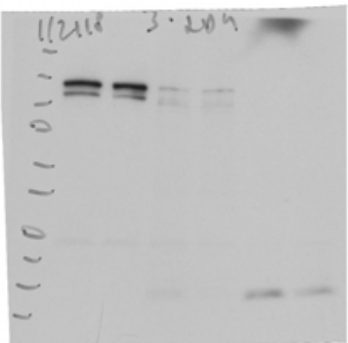

Supp Figure $6 \mathrm{e}$ - cryo2 - control

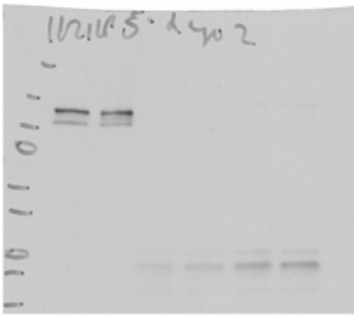

Supp Figure 6d - cryo2 - pronase

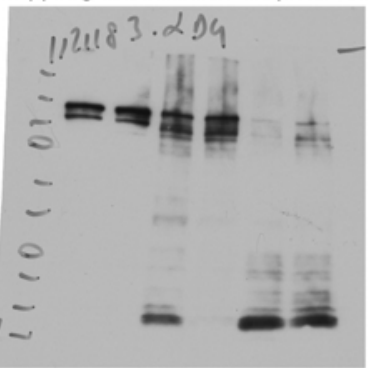

Supp Figure 6d - GAPDH

$=$
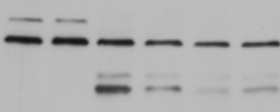

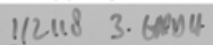

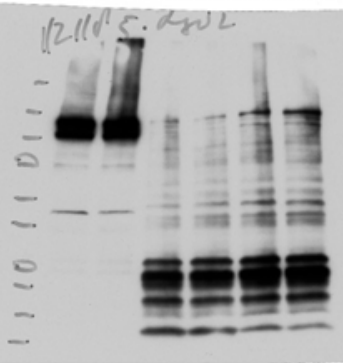

Supp Figure 6e - GAPDH

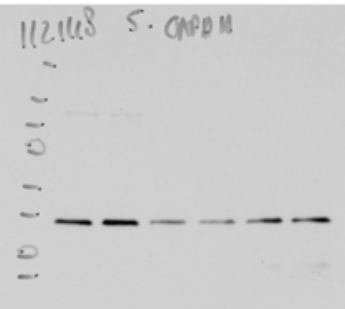




\section{Supplementary Note - Synthetic Procedures}

Photoaffinity probe (PAP) synthesis. The benzophenone PAP (1) design was inspired by our previous work on insulin-secretory NLRP3 inhibitors, whereby we demonstrated that the sulfonyl arene could tolerate large substituents without abrogating NLRP3-inhibitory activity ${ }^{33}$. Compound 1 was synthesised via the 4-step route described below. All commercial regents were used as received, without further purification. All reaction solvents were anhydrous, $\geq 99.9 \%$ purity, purchased from Sigma Aldrich.
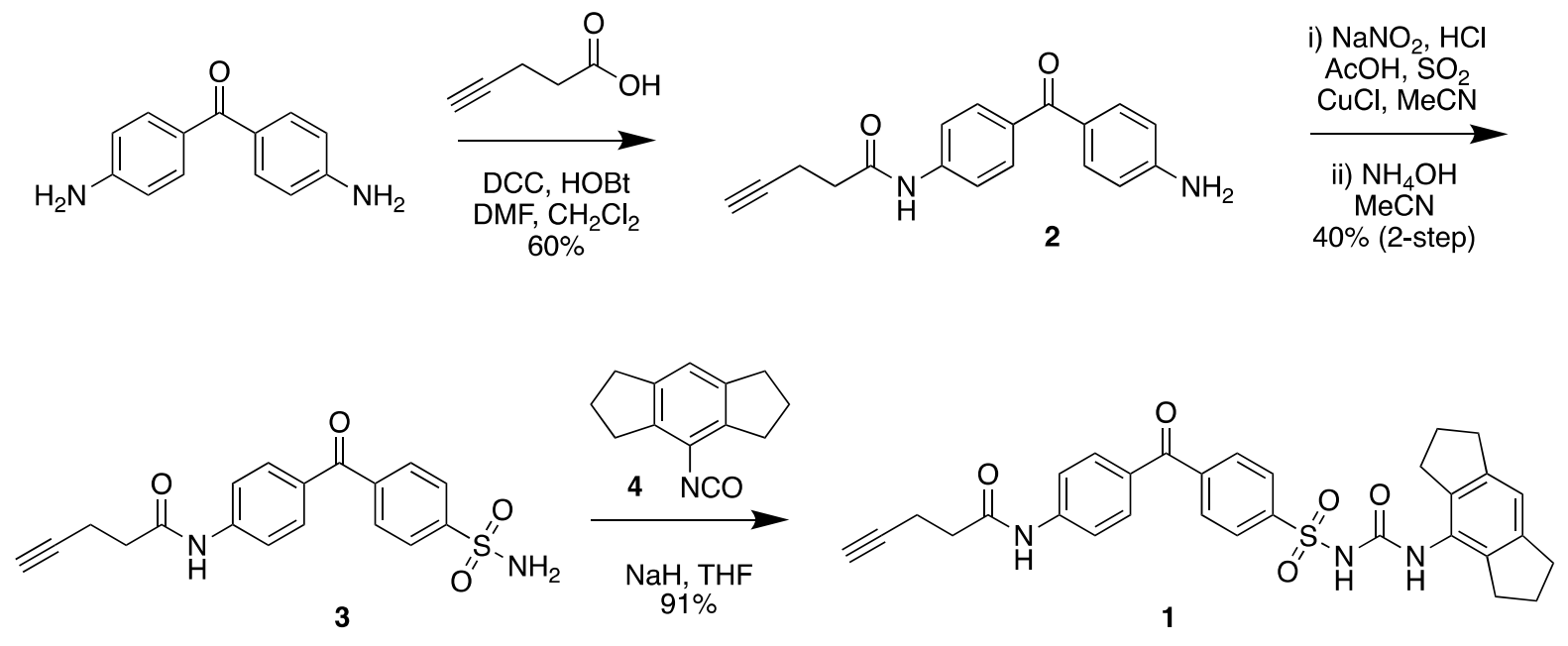

$\mathrm{N}$-(4-(4-Aminobenzoyl)phenyl)pent-4-ynamide (2)<smiles>C#CCCC(=O)Nc1ccc(C(=O)c2ccc(N)cc2)cc1</smiles>

Compound 2 was synthesised using a method described by Tantama et al., with minor changes ${ }^{34}$. To a solution of 4-pentynoic acid $(500 \mathrm{mg}, 5.1 \mathrm{mmol}$, Aldrich, $\geq 95 \%$ purity) in DMF $(10 \mathrm{~mL})$ was added bis(4-aminophenyl)methanone $(1.6 \mathrm{~g}, 7.6 \mathrm{mmol}$, Fluorochem, $\geq 95 \%$ purity) in DMF (10 mL), 1-hydroxybenzotriazole hydrate (HOBt, $690 \mathrm{mg}, 5.1 \mathrm{mmol}$, Advanced Chem Tech, purity not specified) in DMF $(10 \mathrm{~mL})$ and $N, N^{\prime}$-dicyclohexylcarbodiimide (DCC, $1.6 \mathrm{~g}, 7.6 \mathrm{mmol}$, Sigma Aldrich, $\geq 99 \%$ purity) in $\mathrm{CH}_{2} \mathrm{Cl}_{2}(7.6 \mathrm{~mL})$. The mixture was then stirred at room temperature (RT) under nitrogen atmosphere. After $48 \mathrm{~h}$ the reaction was quenched with water $(2 \mathrm{~mL})$ and concentrated in vacuo to give an orange slurry. The product was dissolved in MeCN $(20 \mathrm{~mL})$, filtered and washed with $\mathrm{MeCN}(5 \mathrm{~mL})$. The filtrate was dried in vacuo to afford an orange oil $(1.67 \mathrm{~g})$. The crude product was dissolved in water/MeCN (1:3, $15 \mathrm{~mL}$ ) and purified via $\mathrm{C}_{18}$ medium pressure liquid chromatography (MPLC; gradient elution of $0.1 \%$ formic $\operatorname{acid}_{(\mathrm{aq})}-0.1 \%$ formic acid $\mathrm{MeCN}$ ) to afford the titled product as a pale orange solid $(900 \mathrm{mg}, 60 \%) .{ }^{1} \mathrm{H}$ nuclear magnetic resonance (NMR) $(600$ $\mathrm{MHz}$, Acetone- $\left.d_{6}\right) \delta 9.80(\mathrm{~s}, 1 \mathrm{H}, \mathrm{H}-6), 7.78(\mathrm{~d}, \mathrm{~J}=8.6 \mathrm{~Hz}, 2 \mathrm{H}, \mathrm{H}-9), 7.65$ (d, $J=8.6$ $\mathrm{Hz}, 2 \mathrm{H}, \mathrm{H}-8), 7.59$ (d, J = 8.6 Hz, 2H, H-13), $6.72(\mathrm{~d}, \mathrm{~J}=8.6 \mathrm{~Hz}, 2 \mathrm{H}, \mathrm{H}-14), 5.57$ (s, $2 \mathrm{H}, \mathrm{H}-16), 2.65(\mathrm{t}, \mathrm{J}=7.4 \mathrm{~Hz}, 2 \mathrm{H}, \mathrm{H}-4), 2.54(\mathrm{dt}, \mathrm{J}=7.4,2.6 \mathrm{~Hz}, 2 \mathrm{H}, \mathrm{H}-3), 2.38(\mathrm{t}, \mathrm{J}$ $=2.6 \mathrm{~Hz}, 1 \mathrm{H}, \mathrm{H}-1) .{ }^{13} \mathrm{C} \mathrm{NMR}(151 \mathrm{MHz}$, Acetone-d6) $\delta 193.5$ (C-11), 170.4 (C-5), 153.9 (C-15), 143.0 (C-7), 134.9 (C-10), 133.2 (C-13), 131.3 (C-9), 126.8 (C-12), 119.0 (C-8), 113.7 (C-14), 83.9 (C-2), 70.3 (C-1), 36.7 (C-4), 14.9 (C-3). HRMS-ESI 
$(m / z)$ calculated for $\mathrm{C}_{18} \mathrm{H}_{17} \mathrm{~N}_{2} \mathrm{O}_{2}[\mathrm{M}+\mathrm{H}]^{+}:$293.1285; found: 293.1284. NMR and HRMS data is consistent with literature ${ }^{33}$.

N-(4-(4-Sulfamoylbenzoyl)phenyl)pent-4-ynamide (3)<smiles>C#CCCC(=O)Nc1ccc(C(=O)c2ccc(S(N)(=O)=O)cc2)cc1</smiles>

Compound 3 was synthesised using a method adapted from Hoffman ${ }^{35}$. To prepare the diazonium mixture, a solution of $2(200 \mathrm{mg}, 0.68 \mathrm{mmol})$ in $\mathrm{MeCN}(5 \mathrm{~mL})$ was cooled to $0-5{ }^{\circ} \mathrm{C}$, then treated with acetic acid (550 $\mu \mathrm{L}$, Sigma Aldrich, $\geq 99 \%$ purity), $37 \% \mathrm{HCl}(270 \mu \mathrm{L})$ and $\mathrm{NaNO}_{2}(53 \mathrm{mg}, 0.75 \mathrm{mmol}$, Sigma Aldrich, $\geq 97 \%$ purity) in water $(10 \mu \mathrm{L})$. The resulting mixture was stirred at $0-5{ }^{\circ} \mathrm{C}$ for $30 \mathrm{~min}$. Meanwhile, the chlorosulfonation solution was prepared. $\mathrm{SO}_{2}$, generated from combining $\mathrm{Na}_{2} \mathrm{SO}_{3}$ (8.6 g, $68 \mathrm{mmol}$, Sigma Aldrich $\geq 98 \%), \mathrm{HCl}(20 \mathrm{~mL}$, Sigma Aldrich, 37\% purity) and water $(20 \mathrm{~mL})$, was bubbled through acetic acid $(10 \mathrm{~mL})$ for $20 \mathrm{~min}$ at RT. CuCl (184 $\mathrm{mg}, 1.37 \mathrm{mmol}$, Sigma Aldrich $97 \%$ ) was then added to the $\mathrm{SO}_{2}$ solution, before cooling it to $0-5{ }^{\circ} \mathrm{C}$. The diazonium mixture was then added dropwise over $10 \mathrm{~min}$ at $0-5{ }^{\circ} \mathrm{C}$ and the reaction stirred for a further $50 \mathrm{~min}$ at RT. The reaction mixture was poured onto ice $(20 \mathrm{~g})$ and extracted with EtOAc $(2 \times 40 \mathrm{~mL})$. The organic phases were neutralised with saturated aqueous sodium bicarbonate, washed with water (30 $\mathrm{mL})$ and brine $(30 \mathrm{~mL})$, then dried $\left(\mathrm{MgSO}_{4}\right)$. Removal of the solvent in vacuo afforded a crude sulfonyl chloride as an orange-brown oil (170 mg). The sulfonylchloride, used without further purification, was dissolved in MeCN (4 mL), treated with $\mathrm{NH}_{4} \mathrm{OH}\left(950 \mu \mathrm{L}, 6.84 \mathrm{mmol}, 28-30 \% \mathrm{NH}_{3}\right)$ and stirred at RT for $45 \mathrm{~min}$. Silica chromatography (20-50\% EtOAc in hexanes) was used to afford the titled compound as a pale orange solid $(97 \mathrm{mg}, 40 \%) .{ }^{1} \mathrm{H}$ NMR $\left(600 \mathrm{MHz}\right.$, Acetone- $\left.d_{6}\right) \delta$ 9.59 (s, 1H, H-6), 8.05 (d, J = 8.6 Hz, 2H, H-13), 7.90 (d, J = 8.6 Hz, 2H, H-14), 7.85 $(\mathrm{d}, \mathrm{J}=8.8 \mathrm{~Hz}, 2 \mathrm{H}, \mathrm{H}-9), 7.80(\mathrm{~d}, \mathrm{~J}=8.8 \mathrm{~Hz}, 2 \mathrm{H}, \mathrm{H}-8), 6.78(\mathrm{~s}, 2 \mathrm{H}, \mathrm{H}-16), 2.67$ (t, $\mathrm{J}=$ $7.1 \mathrm{~Hz}, 2 \mathrm{H}, \mathrm{H}-4), 2.57(\mathrm{dt}, \mathrm{J}=7.3,2.7 \mathrm{~Hz}, 2 \mathrm{H}, \mathrm{H}-3), 2.38(\mathrm{t}, \mathrm{J}=2.7 \mathrm{~Hz}, 1 \mathrm{H}, \mathrm{H}-1) .{ }^{13} \mathrm{C}$ NMR (151 MHz, Acetone-d $\left.d_{6}\right) \delta 194.4$ (C-11), 170.7 (C-5), 147.8 (C-15), 144.7 (C-7), 142.0 (C-12), 132.3 (C-10), 132.2 (C-8), 130.7 (C-14), 126.9 (C-13), 119.3 (C-9), $83.8(\mathrm{C}-2), 70.4(\mathrm{C}-1), 36.7(\mathrm{C}-4), 14.8(\mathrm{C}-3)$. HRMS-ESI $(\mathrm{m} / \mathrm{z})$ calculated for $\mathrm{C}_{18} \mathrm{H}_{17} \mathrm{~N}_{2} \mathrm{O}_{4} \mathrm{~S}[\mathrm{M}+\mathrm{H}]^{+}:$357.0904; found 357.0909.

\section{4-Isocyanato-1,2,3,5,6,7-hexahydro-s-indacene (4)}<smiles>O=C=Nc1c2c(cc3c1CCC3)CCC2</smiles>

To a stirred solution of triphosgene $(7.2 \mathrm{~g}, 24.2 \mathrm{mmol}$, Sigma Aldrich, $\geq 98 \%$ purity) in THF $(20 \mathrm{~mL})$ was added the 1,2,3,5,6,7-hexahydro-s-indacen-4-amine (4.2 g, 24.2 mmol, Advanced Chemical Intermediates, $\geq 95 \%$ purity) in THF (20 mL) dropwise. Triethylamine $(6.7 \mathrm{~mL}, 48 \mathrm{mmol}$, Sigma Aldrich, $\geq 99 \%$ purity) in THF (20 mL) was added dropwise. The mixture was stirred at RT for $3 \mathrm{~h}$, monitoring with TLC. The solid was removed by filtration and the filtrate was concentrated in vacuo. The resulting oil was triturated with petroleum ether $(60 \mathrm{~mL})$ and the solid was removed by filtration. The filtrate was concentrated in vacuo to give a golden oil. The crude 
was purified by rapid filtration through silica, with petroleum ether as solvent, to give the title compound as a colourless oil that crystallised upon cooling ( $4.3 \mathrm{~g}, 91 \%)$. The isocyanate was used immediately in the next synthetic step. ${ }^{1} \mathrm{H}$ NMR $(600 \mathrm{MHz}$, $\left.\mathrm{CDCl}_{3}\right) \delta 6.93(\mathrm{~s}, 1 \mathrm{H}, \mathrm{H}-5), 2.87(\mathrm{q}, \mathrm{J}=7.4 \mathrm{~Hz}, 8 \mathrm{H}, \mathrm{H}-6$ and $\mathrm{H}-8), 2.10$ (p, J = $7.4 \mathrm{~Hz}$, $4 \mathrm{H}, \mathrm{H}-7) .{ }^{13} \mathrm{C}$ NMR $\left(151 \mathrm{MHz}, \mathrm{CDCl}_{3}\right) \delta 144.4$ (C-4), 136.7 (C-3), 125.7 (C-2), 125.4 (C-1), 117.9 (C-5), 33.1 (C-6), 30.2 (C-8), 25.3 (C-7). NMR data is consistent with the literature ${ }^{36}$. HRMS was not conducted as the compound is unstable and does not ionise well. The identity of the compound was further confirmed by the successful reaction to form the desired sulfonylurea (1) which has the desired HRMS ion.

$N-(4-(4-(N-((1,2,3,5,6,7-H e x a h y d r o-s-i n d a c e n-4-$

yl)carbamoyl)sulfamoyl)benzoyl)phenyl)pent-4-ynamide (1)<smiles>C#CCCC(=O)Nc1ccc(C(=O)c2ccc(S(=O)(=O)NC(=O)Nc3c4c(cc5c3CCC5)CCC4)cc2)cc1</smiles>

Compound 1 was synthesised using a method adapted from Dombroski \& Eggler ${ }^{37}$. A solution of sulfonamide $3(40 \mathrm{mg}, 0.11 \mathrm{mmol})$ in THF $(4 \mathrm{~mL})$ at $0{ }^{\circ} \mathrm{C}$ was treated with $\mathrm{NaH}(4.0 \mathrm{mg}, 0.17 \mathrm{mmol}$, Sigma Aldrich, $60 \%$ dispersion in oil,) and stirred at RT under reduced pressure for $15 \mathrm{~min}$. Once effervescence ceased, the orange solution was cooled to $0{ }^{\circ} \mathrm{C}$ and treated with solution of isocyanate 4 (22 $\mathrm{mg}, 0.11$ $\mathrm{mmol})$ in THF (1 mL). The reaction was stirred at RT for $20 \mathrm{~h}$ before drying under a steam of nitrogen. The crude reaction mixture was purified via $\mathrm{C}_{18}$ MPLC (gradient elution of $0.05 \%$ formic $\operatorname{acid}_{(\mathrm{aq})}-\mathrm{MeCN}$, whereby fractions were neutralised with an equivalent volume of $10 \mathrm{mM}$ ammonium bicarbonate $(\mathrm{aq})$ ). Lyophilisation of the appropriate fractions afforded a white solid $(55.6 \mathrm{mg}, 91 \%) .{ }^{1} \mathrm{H} \mathrm{NMR}(600 \mathrm{MHz}$, DMSO-d $)) \delta 10.97$ (br s, 1H, H-16) 10.41 (s, 1H, H-6), $8.04\left(\mathrm{~m}, 3 \mathrm{H}, \mathrm{H}-13\right.$ and $\left.\mathrm{H}-1^{\prime}\right)$, $7.84(\mathrm{~d}, \mathrm{~J}=8.3 \mathrm{~Hz}, 2 \mathrm{H}, \mathrm{H}-14), 7.79(\mathrm{~d}, \mathrm{~J}=8.8 \mathrm{~Hz}, 2 \mathrm{H}, \mathrm{H}-9), 7.74(\mathrm{~d}, \mathrm{~J}=8.8 \mathrm{~Hz}, 2 \mathrm{H}$, $\mathrm{H}-8), 6.89$ (s, 1H, H-5'), 2.81 (t, J = 2.6 Hz, 1H, H-1), 2.77 (t, J = 7.4 Hz, 4H, H-6'), $2.60-2.54\left(\mathrm{~m}, 6 \mathrm{H}, \mathrm{H}-4\right.$ and $\left.\mathrm{H}-8^{\prime}\right), 2.49-2.46(\mathrm{~m}, 2 \mathrm{H}, \mathrm{H}-3), 1.91$ (p, J = 7.4 Hz, 4H, H-7'). ${ }^{13} \mathrm{C}$ NMR (151 MHz, DMSO-d6) ס 193.5 (C-11), 170.0 (C-5), 149.2 (C-17), 143.6 (C-7), 142.7 (C- 4'), 142.8 (C-15), 140.6 (C-12), 137.0 (C-3'), 131.2 (C-9), 130.8 (C-2'), 130.5 (C-10), 129.3 (C-13), 127.1 (C-14), 118.2 (C-8), 117.3 (C-5'), 83.3 (C-2), 71.5 (C-1), 35.2 (C-4), 32.4 (C-6'), 30.0 (C-8'), 24.9 (C-7'), 13.8 (C-3). HRMS-ESI $(\mathrm{m} / \mathrm{z})$ calculated $\mathrm{C}_{31} \mathrm{H}_{30} \mathrm{~N}_{3} \mathrm{O}_{5} \mathrm{~S}[\mathrm{M}+\mathrm{H}]^{+}:$556.1901; found 556.1891.

NMR spectra related to photoaffinity probes synthesis. Each compound was characterised using ${ }^{1} \mathrm{H}$ NMR and ${ }^{13} \mathrm{C}$ NMR. Spectra were recorded using a Bruker Avance $600 \mathrm{MHz}$ spectrometer operating at $600 \mathrm{MHz}$ for ${ }^{1} \mathrm{H}$ NMR and $151 \mathrm{MHz}$ for ${ }^{13} \mathrm{C}$ NMR. ${ }^{1} \mathrm{H}$ and ${ }^{13} \mathrm{C}$ chemical shifts are referenced to tetramethylsilane $(\delta=0 \mathrm{ppm})$, where present, or to the appropriate NMR solvent. 


\section{$2{ }^{1} \mathrm{H}$ NMR}

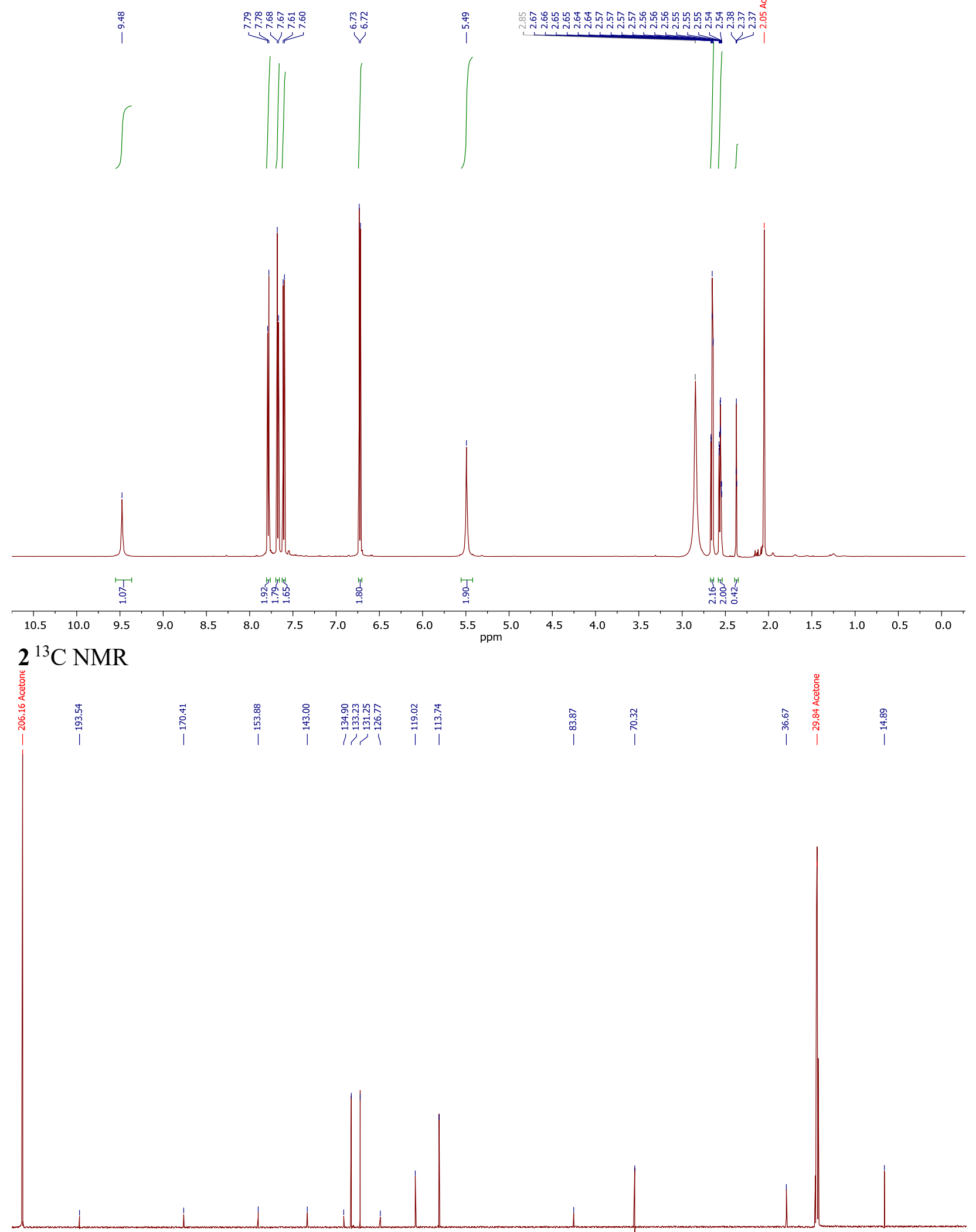

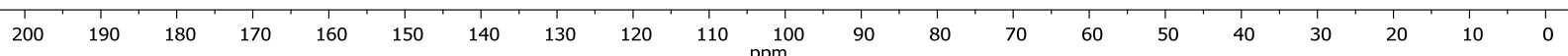


$3{ }^{1} \mathrm{H}$ NMR

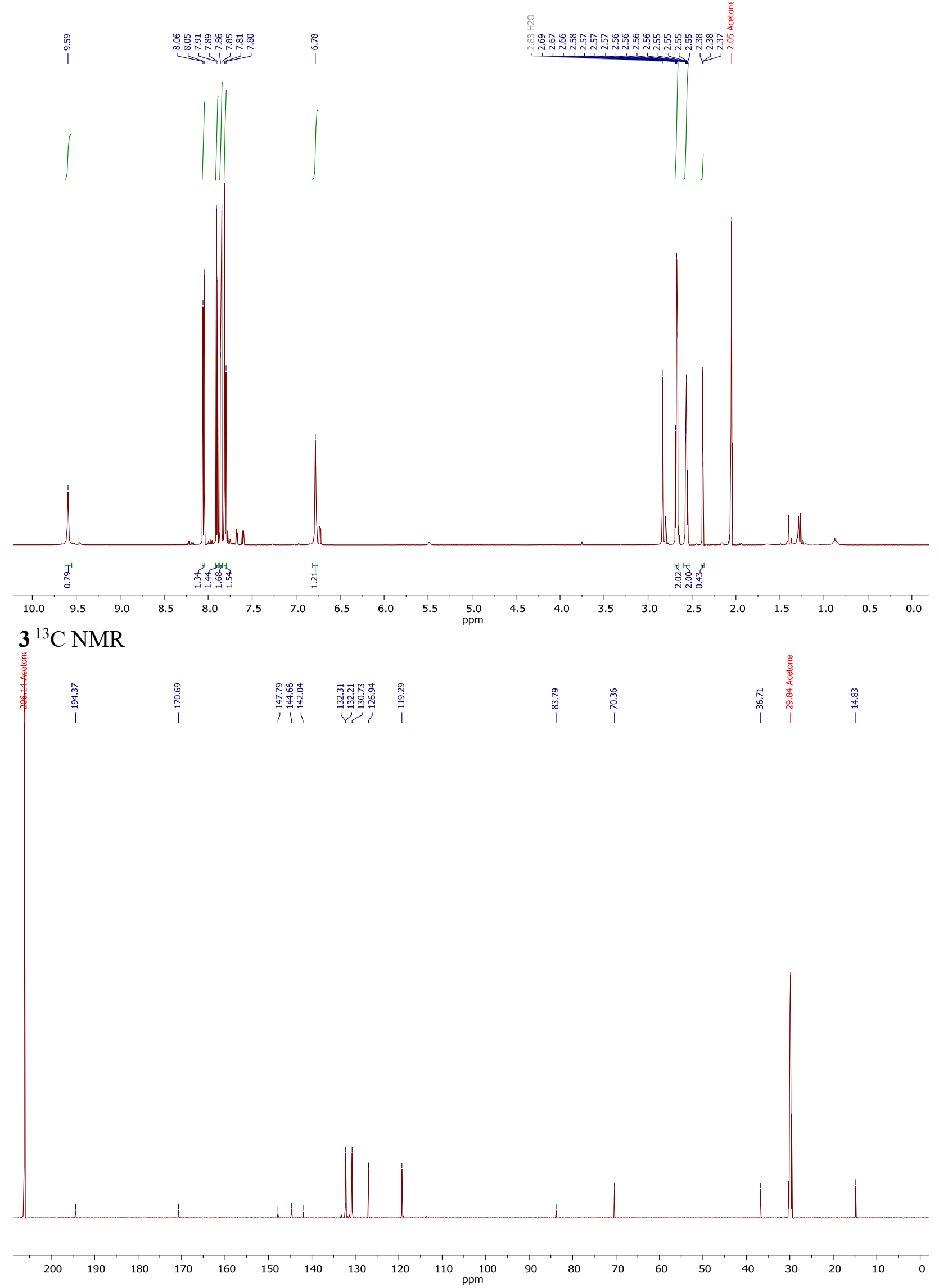


$4{ }^{1} \mathrm{H}$ NMR

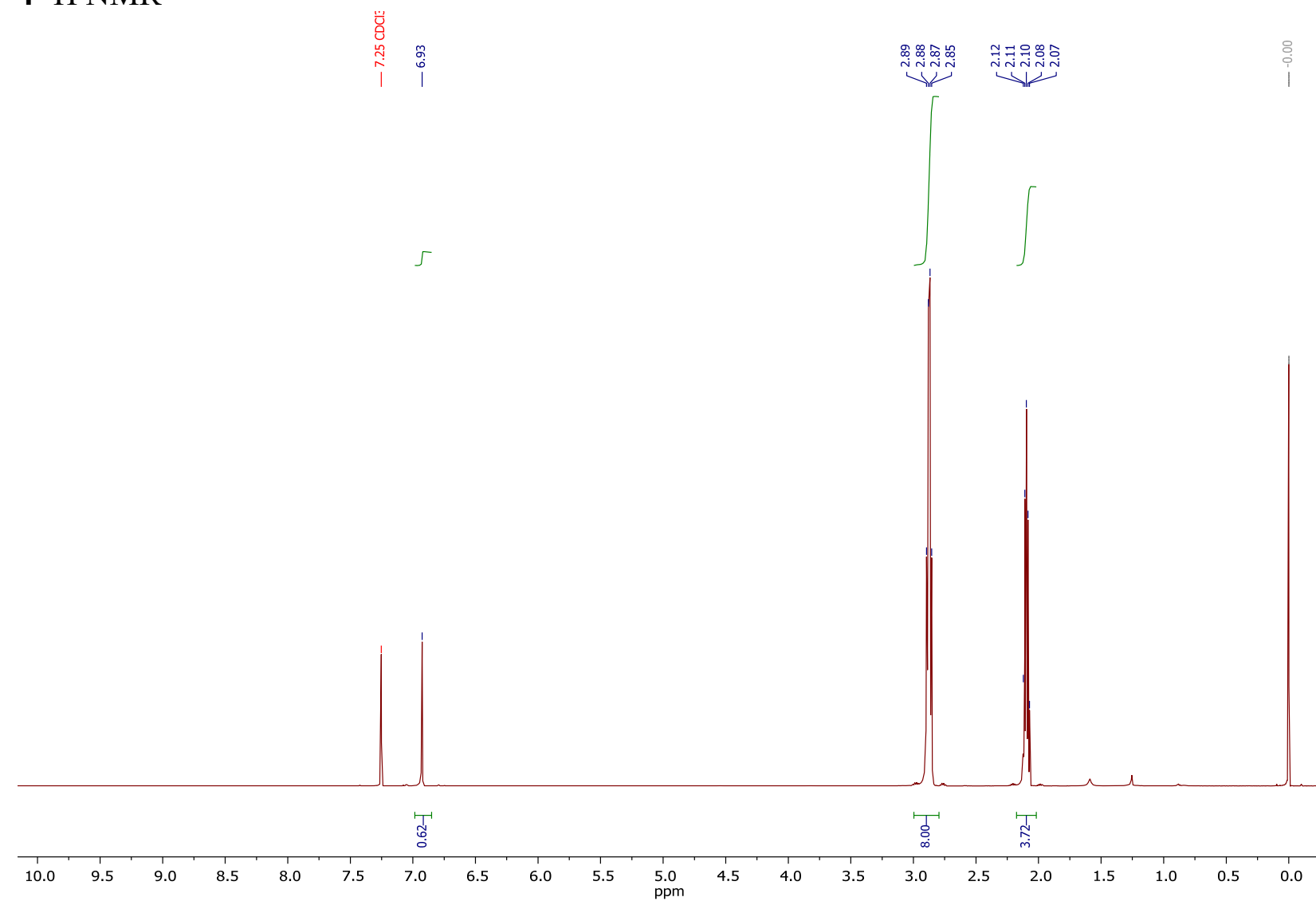
$4{ }^{13} \mathrm{C}$ NMR

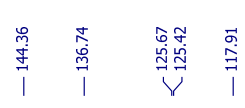

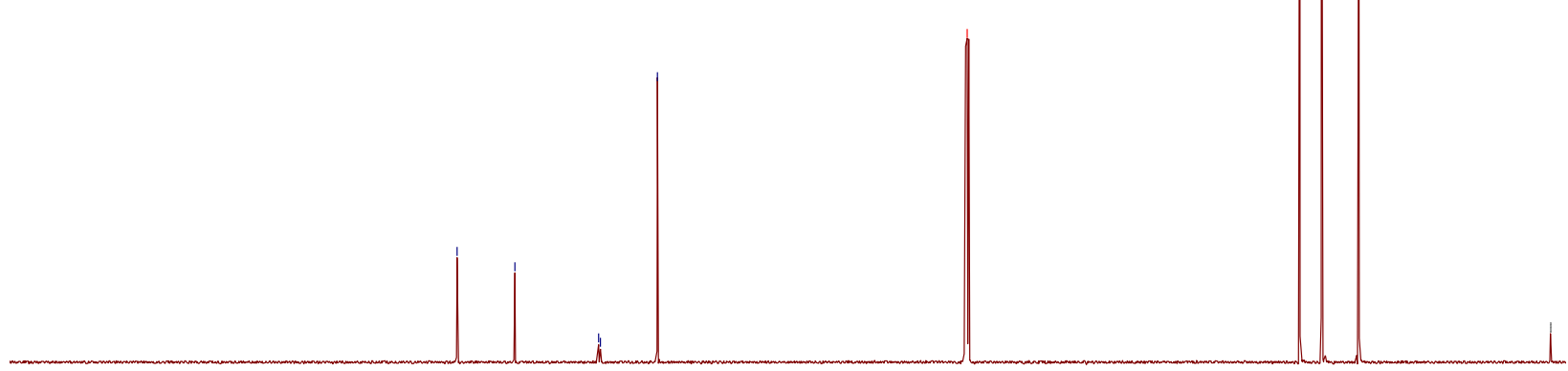

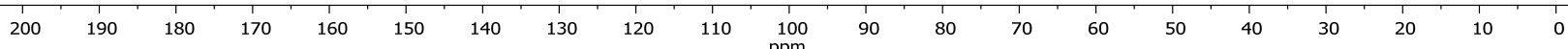




\section{$\mathbf{1}^{1} \mathrm{H}$ NMR}

i

$\underbrace{\text { ind }}$

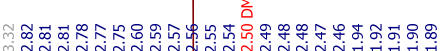

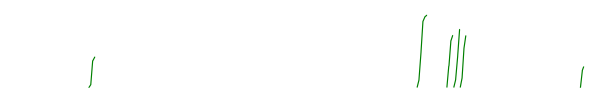

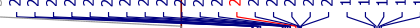

बे
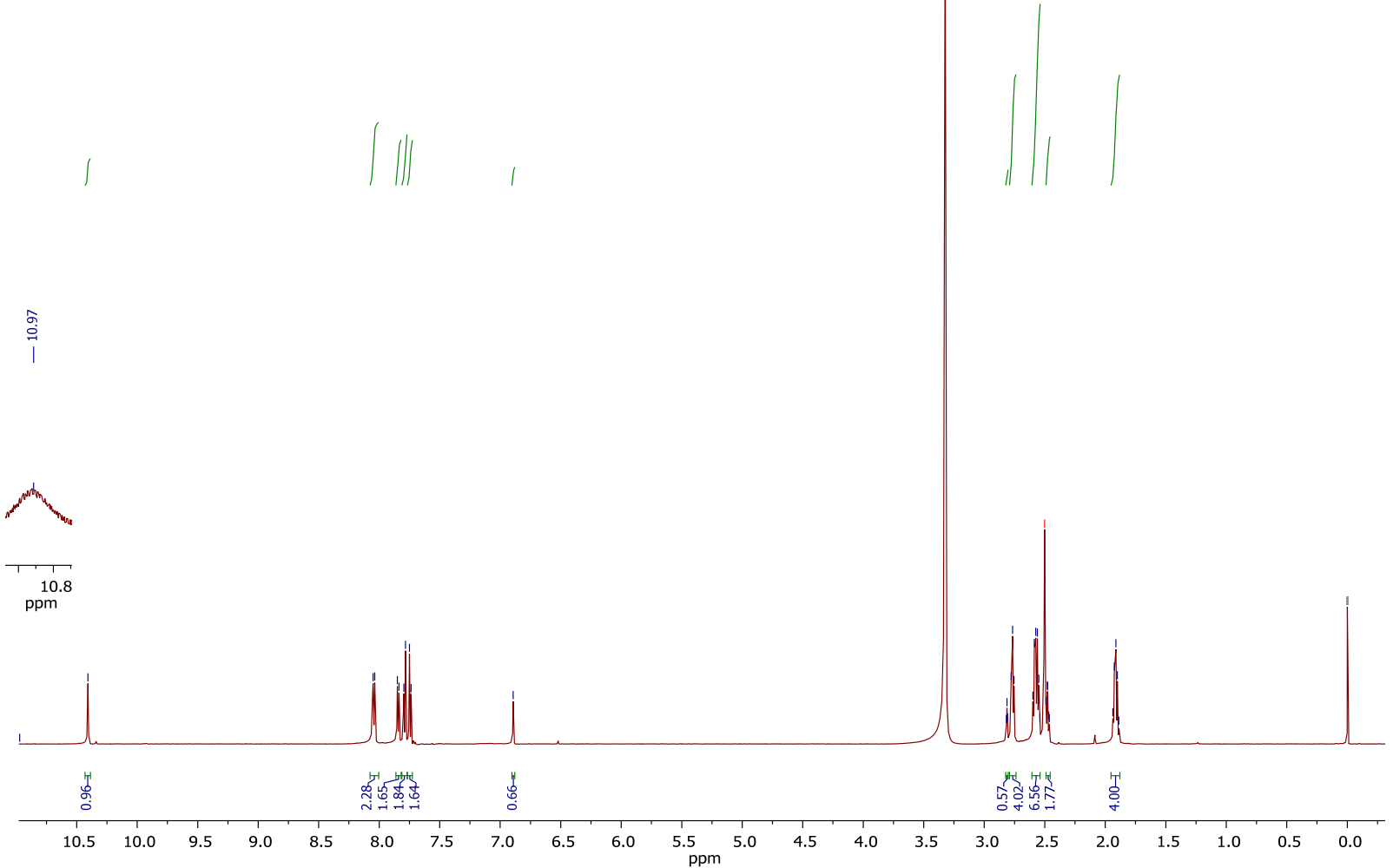

$1^{13} \mathrm{C}$ NMR

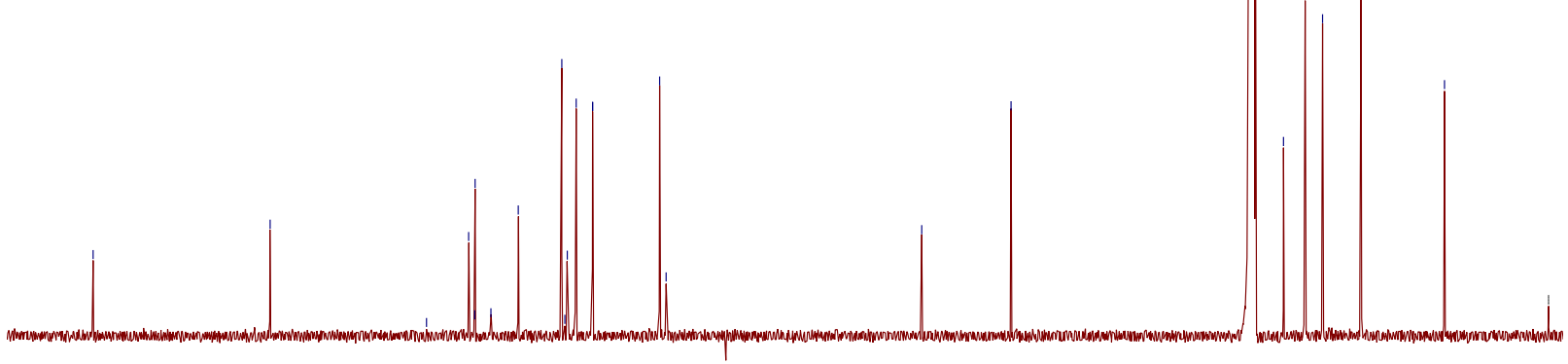

$200 \quad 190$

$180 \quad 170$

$160 \quad 150$

$\begin{array}{llll}140 & 130 & 120 & 1\end{array}$ 


\section{Supplemental Note References}

33. Hill, J.R., et al. ChemMedChem 12, 1449-1457 (2017).

34. Tantama, M., Lin, W.C. \& Licht, S. J Am Chem Soc 130, 15766-15767 (2008).

35. Hoffman, R.V. Org Synth 60, 121-126 (1981).

36. Urban, F. J. et al. Synth. Commun. 33, 2029-2043 (2003).

37. Dombroski, M.A., Eggler, J. F. (1998). International Application No.: PCT/IB1997/001603. 